\title{
HUB LOCATION PROBLEM IN ROUND-TRIP SERVICE APPLICATIONS
}

\author{
Omar Kemmar ${ }^{1, *}$, Karim Bouamrane ${ }^{1}$ and Shahin Gelareh ${ }^{2}$
}

\begin{abstract}
In this paper, we introduce a new hub-and-spoke structure for service networks based on round-trips as practiced by some transport service providers. This problem is a variant of Uncapacitated Hub Location Problem wherein the spoke nodes allocated to a hub node form round-trips (cycles) starting from and ending to the hub node. This problem is motivated by two real-life practices in logistics wherein runaway nodes and runaway connections with their associated economies of scale were foreseen to increase redundancy in the network. We propose a mixed integer linear programming mathematical model with exponential number of constraints. In addition to the separation routines for separating from among exponential constraints, we propose a hyper-heuristic based on reinforcement learning and its comparable counterpart as a variable neighborhood search. Our extensive computational experiments confirm efficiency of the proposed approaches.
\end{abstract}

Mathematics Subject Classification. 68T20, 90C59, 90C27, 90B80, 90C35, 90C05, 90C11.

Received January 31, 2020. Accepted October 31, 2020.

\section{INTRODUCTION}

While hub-and-spoke operations have almost always been practiced in the modern transportation industry, in certain circumstances, these structures are the only possible way ahead. This work has been motivated by two consultancy projects that have been carried out over the last 5 years: a set of maritime projects and an aid distribution problem arising in the context of Syrian refugees in Lebanon.

We are dealing with structures depicted in Figure 1 (see [21]), which frequently appear in various and sometimes very different areas including supply chain logistics as well as telecommunications.

However, this structure has some weaknesses, as every potential failure or disruption can render an important part of the network unreachable. In the following, we point out two cases that we have encountered in our collaboration with the stakeholders from the industry.

Liner shipping industry. In liner shipping wherein vessels operate round-trips, this notion has received much more attention. About 80 per cent of the world trade by volume and more than 70 per cent of world trade in value is carried by sea, making liner shipping the basis of world trade [46]. Liner shipping operates on hub-and-spoke structures where major terminals are considered as global or regional hubs and the smaller

Keywords. Hub location problem, liner shipping, runaway node, branch-and-cut, hyper-heuristic, variable neighborhood search, reinforcement learning, $k$-means.

1 Laboratoire d'informatique d'Oran (LIO), Université Oran 1, BP 1524 EL Mnaouer Oran, Algeria.

2 Département Réseaux et Télécommunications, Université d'Artois, F-62400 Béthune, France.

*Corresponding author: Omke1941@hotmail.com, omke1993@gmail.com 


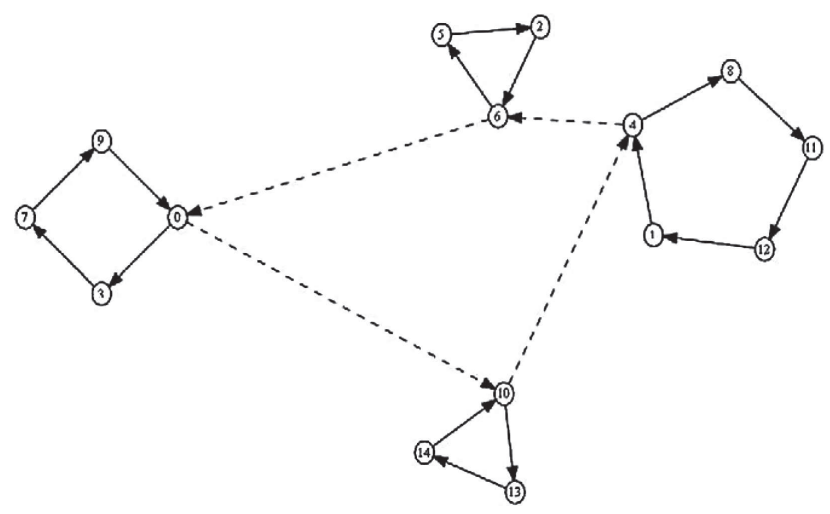

FiguRE 1. A typical hub-and-spoke based round-trip service network.

ports are the spoke/feeder ports. Almost a decade ago, when the extremely costly and spacious mega-vessels of $18000 \mathrm{TEU}$ (twenty-foot equivalent) such as Emma Maersk have emerged and were deployed, both ports and liner operators were really concerned. The string managers (those in charge of designing seasonal roundtrip service network in shipping companies) were worried that such costly and huge vessels may have to be deployed under-utilized (at least during some periods) without being able to efficiently exploit their economies of scale. The port operators or major terminals were worried of being now exposed to a much higher risk should any possible disruptions occur. If such a disruption happens and delays go beyond the normal and expected turnaround time for the vessels (which would increase operation costs for liners), it would causes dissatisfaction for the liners and jeopardize the ports competitiveness in their business. Such large vessels could not call many ports due to the draught requirement and moreover, many European ports did not have the capacity (not even potential for physical expansion) to serve such huge vessels. The solution was mainly in the hand of network operators rather than hub operators, to introduce sufficient redundancy such that a reasonable part of the risks could be mitigated. This could be achieved by providing possibility of cross-rotation escape operation through some nominated ports. One way to achieve this was to make sure that the feeder round-trips do not rely on (not monopolized by) one and only one hub port and if the single hub fails (due to strikes, threats, etc.), the operation can still be re-directed through runaway connections at a minimal overhead operational cost.

Distribution of humanitarian aids. A very recent case we have recently worked on is related to distribution of humanitarian aids among the UNHCR-recognized displaced Syrians who fled out of the war situation and scattered all across Lebanon as one of the major host countries. A central depot in Beirut suburbs, supplies a set of hub locations identified among the demand nodes. Cycles are formed starting from every hub locations, visiting a set of spoke demand points (the villages wherein refugees are residing) and returning to the depot on the same day (see Fig. 2). For some domestic reasons, it is very likely that a hub node becomes unavailable at anytime without any prior notice. This can even happens after it receives the supply for spoke nodes allocated to it in which case new supply must be sent from the main depot but via a different runaway node and runaway connection.

We need to introduce some redundancy in the hub-and-spoke structure in Figures 1 and 3 in such a way that in an (un)likely event of disruption in the hub-level elements, the service provision remains possible. Such a redundancy can be achieved by providing alternative paths for spoke nodes on the cycle (feeder) of a given hub node. We achieve this by connecting cycles via a third type of nodes we refer to as runaway nodes. These nodes do not necessarily incorporate very sophisticated and expensive hub-type facilities, yet the infrastructure is upgraded to outperform spoke nodes and enable them to process larger volumes in the case of necessity. 


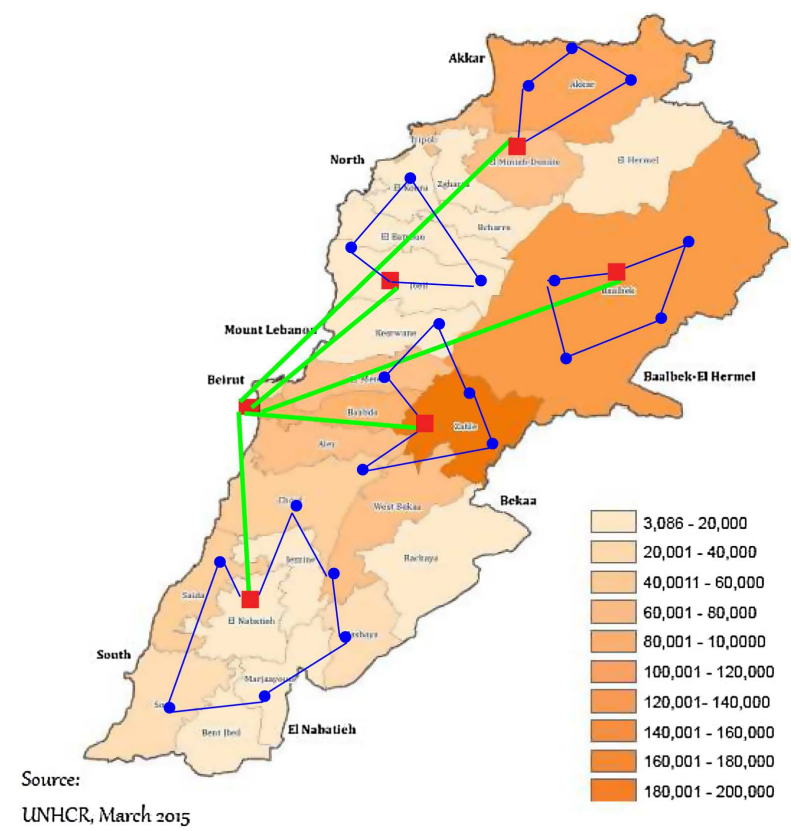

(a)

FIGURE 2. Network structure for a hub-and-spoke structure distribution network of humanitarian aids in Lebanon. Source: Displaced Syrian distribution by CAZA, Lebanese Government.

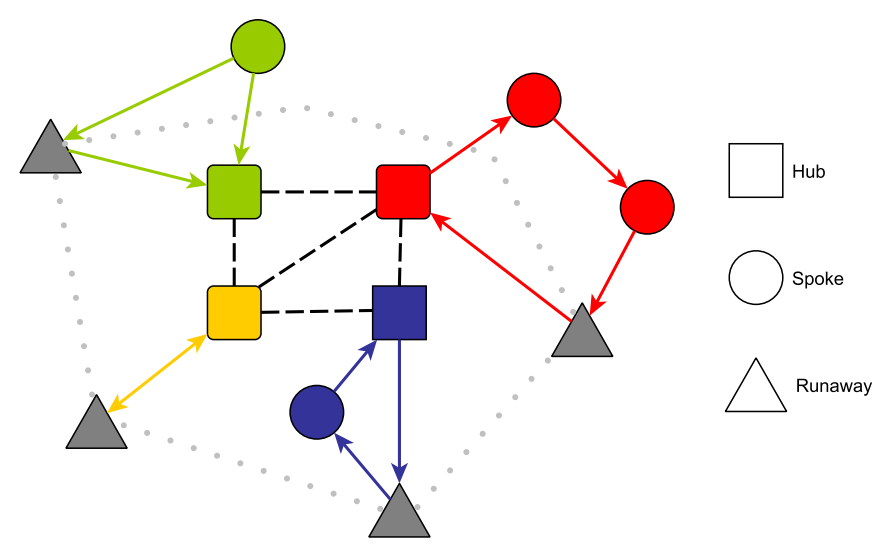

FiguRE 3. A typical structure of network in a runaway hub-and-spoke structure.

\subsection{Literature review}

This work can be considered as a variant of the uncapacitated single allocation hub location problem (USAHLP). In the following, we review some of the closely related contributions in the literature, with particular attributes: (1) single allocation scheme, (2) cyclic spoke-level sub-networks, and (3) solution approaches based on (meta-)heuristics and hyper-heuristics. 
Danach et al. [16] proposed a Mixed Integer Programming (MIP) formulation, a Lagrangian relaxation and a hyper-heuristic for a hub location and routing problem. In this study, the single allocation scheme is considered where the capacity is defined on the volume of flow circulating on a spoke-level route that needs to respect the capacity of transporters available on it.

Azizi [2] introduced a MIP formulation and a Particle Swarm Optimization (PSO) algorithm for the Uncapacitated Single Allocation p-hub Location Problem under risk of hubs disruption. The author constructs networks in which every single demand point has a backup hub to be served from in case of disruption.

Zhong et al. [52] tackled the hierarchical hub location model and proposed a MIP formulation for the problem with hub capacity constraints as well as a hybrid meta-heuristic (genetic algorithm and tabu search).

A two-stage formulation for reliable Single Allocation Hub Location Problem was introduced in Rostami et al. [42] with a Benders decomposition approach to solve large scale instances. In this study, whenever a hub breaks down, its corresponding flow is rerouted via a single backup hub.

Monemi and Gelareh [33] proposed a 2-index integer programming (IP) formulation and a branch-and-cut algorithm with some classes of valid inequalities for the Ring Spur Assignment Problem introduced by Carroll et al. [9]. This problem arises in the design of next-generation telecommunications networks but shares some common features with this work.

A 2-index model and a branch-and-cut algorithm based on Benders decomposition are given in Gelareh et al. [22] for the Bounded Cardinality Capacitated Hub Routing Problem (BCCHRP) with route capacity constraints.

In Chaharsooghi et al. [11], the reliable uncapacitated multiple allocation hub location problem under hub disruptions is considered. The author proposed a two-stage stochastic model and an adaptive large neighborhood search meta-heuristic as a non-exact approach. In this problem, whenever a hub fails, the spokes allocated to that hub, are either reallocated to other hubs that are still working or a penalty is paid in the case they do not receive any service due to the high reallocation costs.

Contreras et al. [12] presented a MIP formulation and a branch-and-cut algorithm for the Cycle Hub Location Problem (CHLP). A greedy randomized adaptive search procedure (GRASP) is developed to obtain feasible solutions for large-scale instances of the CHLP.

Rodriguez-Martin et al. [41] proposed a branch-and-cut algorithm for the problem of designing a two level network where the upper level consists of a backbone ring network connecting the hub nodes, and the lower level is formed by access ring networks that connect the spoke nodes to the hub nodes. It is a purely location problem and does not incorporate any flow.

Mohammadi et al. [32] proposed a bi-objective mixed-integer non-linear programming and an evolutionary algorithm for the Single Allocation p-hub Center-Median Problem under data uncertainty, where the objective is to obtain a reliable network. Interested readers are also referred to the recent network reliability studies: Yahyaei et al. [48], Zhalechian et al. [51] and Cardoso et al. [7].

In Martins de Sá et al. [29] a Benders decomposition algorithm and several metaheuristics were proposed for the Hub Line Location Problem (HLLP) introduced in Martins de Sá et al. [30]. This problem is tailored for public transportation systems.

The hub location and routing problem is studied in Rodriguez-Martin et al. [40]. A MIP formulation and a branch-and-cut algorithm are proposed. In this work, the capacity constraint is defined in terms of number of spokes per cycle, and the number of hubs is assumed to be an exogenous information.

Gelareh et al. [21] proposed a hub-and-spoke structure with one central hub cycle and spoke-level (feeder) cycles attached to every hub node. They use Lagrangian decomposition approach equipped with a Lagrangian heuristic.

Yang et al. [50] proposed a hybrid particle swarm optimization (PSO) algorithm for the $p$-Hub Center Problem in fuzzy environments by combining PSO, genetic operators and a local search (LS).

Alumur et al. [1] proposed a MIP for a hierarchical multi-modal hub location problem, where two types of hub nodes and hub edges are considered (for ground and air transportation) and a time definite delivery service. 
Gelareh and Nickel [20] proposed a MIP with a Benders decomposition method and a greedy heuristic to solve the Uncapacitated Multiple Allocation Hub Location Problem adapted for urban transport and liner shipping network design.

Çetiner et al. [10] proposed an iterative two stages heuristic, which firstly locates the hub nodes and then design routes using traveling salesman problem heuristics. The Turkish postal delivery system data is used as a case study.

A Reliable p-hub Location Problem was proposed in Kim and O'Kelly [26] for telecommunication networks, where two mathematical model formulations are given considering the single and multiple assignment schemes.

In Berman et al. [3], a model is proposed in which, given $n$ nodes, the objective is to locate $p$ helicopter pads and one facility (hospital) to minimize the total time (minisum) or the maximum time (minimax).

Yaman et al. [49] proposed a minimax mathematical model for ground-based cargo delivery system with stopovers for the latest arrival hub location problem.

A local search approach and different meta-heuristic algorithms were proposed in Carello et al. [8] for the capacitated single allocation hub location problem (CSAHLP) where the hubs are transit nodes and the spokes are access nodes.

A branch-and-bound procedure was proposed in Ebery et al. [18] for the capacitated multiple allocation hub location problem applied to postal networks together with an efficient heuristic algorithm using shortest paths to obtain the upper bound.

Campbell [6] presented the first linear programming formulations for Multiple/Single Allocation Uncapacitated/Capacitated Hub Location Problems. This work has introduced a fundamental set of hub location problems that have served as a building block for a lot of research works. Skorin-Kapov et al. [44] proposed an improvement to the best-known MIP formulation for the Single Allocation p-hub Median Problem (SApHMP).

Kuby and Gray [27] developed a MIP formulation to design the least cost single-hub air network assuming that the hub location is predetermined.

O'Kelly [35] introduced single allocation hub location problem (SAHLP) with fixed costs making the number of hubs an endogenous part of the problem and proposed a quadratic integer formulation. Campbell [5] introduced the first model for the multiple allocation problem.

For most part, the works tackling Hub Location Problems in the literature consider the standard hub and spoke network structures. In addition, most of the non-exact approaches proposed in the previous studies are generally heuristics and meta-heuristics, leaving great potential of the hyper-heuristic approaches almost untouched in this context.

This paper proposes an extension to the work in Danach et al. [16] and therefore shares some elements. However, our work is distinguished from Danach et al. [16] as follows: in Danach et al. [16], the hub-level network is a complete subgraph, while in here, it is an endogenous part of the problem and not necessarily a complete subgraph. In the aforementioned work, there are two sorts of nodes and connections (spoke and hub nodes), while in this study we define an additional type of node/connection and we use the conventional term from practice to refer to it as runaway. Runaway nodes and edges (the edges connecting runaway nodes) provide alternative paths and augment the redundancy to meet the origin-destination demands. Finally, in Danach et al. [16], every origin-destination demand passes through the hub-level network while this is not necessary the case in the current work.

The main features of the most related contributions in the literature are summarized in Table 1.

\subsection{Contribution and scope}

The problem in this paper is motivated by a couple of real-life cases wherein the spoke-level network structure resembles the one depicted in Figures 2 and 3. In the real practice, we seek to upgrade such structures to introduce more redundancy through runaway nodes such as the one in Figure 3. The dashed lines (runaway connections) connecting runaway nodes (nodes that have attributes somewhere between being a sophisticated hub node and a simple spoke node) lying on the spoke-level round-trips introduce some level of redundancy and cope with potential failures (strikes, unforeseen threats, etc.) at the hub nodes or the connections to/from 
TABLE 1. A summary of the relevant contributions in the literature.

\begin{tabular}{|c|c|c|c|c|c|}
\hline Work & Alloc. & Num. hubs & Objective & Capacity & Solution method \\
\hline Danach et al. [16] & SA & Exogenous & $\begin{array}{l}\text { Time (transit }+ \\
\text { transshipment) }\end{array}$ & Yes & $\begin{array}{l}\text { MIP + Lagrangian relax- } \\
\text { ation + Hyper-heuristic }\end{array}$ \\
\hline Zhong et al. [52] & SA & Endogenous & Cost & Yes & MIP + Meta-heuristic \\
\hline Contreras et al. [12] & SA & Exogenous & Cost & No & $\begin{array}{l}\text { MIP + Branch-and-cut }+ \\
\text { Meta-heuristic }\end{array}$ \\
\hline Rodriguez-Martin et al. [41] & SA & Endogenous & Cost & $\begin{array}{l}\leq q \text { spokes } \\
\text { per access ring } \\
+1 \leq \text { access } \\
\text { rings } \leq k \text { per } \\
\text { hub }\end{array}$ & MIP + Branch-and-cut \\
\hline Gelareh et al. [22] & SA & $q=3 \leq \ldots \leq p$ & $\begin{array}{l}\text { Time (transit }+ \\
\text { transshipment) }\end{array}$ & Yes & $\begin{array}{l}\text { MIP }+ \text { Branch-and-cut }+ \\
\text { Benders decomposition }\end{array}$ \\
\hline Rodriguez-Martin et al. [40] & SA & Exogenous & Cost & $\begin{array}{l}\leq q \text { spokes per } \\
\text { cycle }\end{array}$ & MIP + Branch-and-cut \\
\hline Gelareh et al. [21] & SA & Exogenous & Cost & $\begin{array}{l}\geq q \text { spokes per } \\
\text { cycle }\end{array}$ & $\begin{array}{l}\text { MIP + Lagrangian decom- } \\
\text { position based heuristic }\end{array}$ \\
\hline Çetiner et al. [10] & MA & Endogenous & $\begin{array}{l}\text { Cost }+ \text { Num. of } \\
\text { vehicles }\end{array}$ & No & Heuristic \\
\hline Current work & SA & Exogenous & Cost & No & $\begin{array}{l}\text { MIP + Meta-heuristic + } \\
\text { Hyper-heuristic }\end{array}$ \\
\hline
\end{tabular}

the hub nodes. In contrast to a backup hub node and sophisticated hub edges (connecting such backup node to the hub-level network) which are expensive to setup and maintain, the runway nodes are some enhancements to the normal spoke nodes and are only connected to one or two peer runaway nodes using connections that are somewhere between the performance of a hub edge and a spoke edge, making it much cheaper to operate and maintain until the disruption is over. Moreover, when the hub-level network is targeted by a disruptive occurrence and becomes temporarily unavailable, this notion provides excess capacity somewhere outside the focus of disruption and in a decentralized fashion - i.e. at the spoke level network. We have carried out a thorough investigation of the matter in two cases from the real practice and a close collaboration with the relevant decision makers from the sectors. From the theoretical and modeling point of view, this model opens a new perspective in including redundancy in the hub-and-spoke structures and generalizes some of the previous work (including our work in [21]). We propose the first (exponential) mixed integer programming formulation for this problem, an efficient separation routine to separate from among the exponentially many constraints, and an efficient heuristic-selection hyper-heuristic delivering high quality solutions in a very reasonable time. Extensive computational experiments on randomly generated instances of various sizes, confirm computational efficiency of the proposed solution framework and the viability of the approach.

This paper is organized as follows: The problem is formally described in Section 2 and a mathematical model with an exponential number of constraints is proposed in Section 3. Section 4 provides a detailed description and elaborates on the components of the hyper-heuristic approach (initial solution, selection method, lowlevel heuristics and the main procedure), the metaheuristic and the separation routine. Section 5 reports and discusses the results of our computational experiments. In Section 6, we summarize, draw conclusions and provide suggestions for further research directions.

\section{Problem statement}

The $p$-Hub Location Problem with Runaway ( $p$ HLPwR) (Fig. 3) can be formally described as in the following: Given a set of nodes $V$ where $|V|=n$, a cost matrix $C$ where $c_{i j}$ is the cost per unit of flow on the edge $i$ to $j$, a flow/demand matrix $W$ where $w_{i j}$ is the flow to be sent from $i$ to $j$ and the fixed costs of setting up 
hub nodes and runaway nodes. The fixed costs of setting up hub and runaway nodes, hub edges, spoke arcs and runaway connections are denoted by $F_{k}, G_{k}, I_{k l}, S_{k l}$ and $T_{k l}$, respectively. The problem is to find $p$ nodes called hub nodes and establish the hub-level network using hub edges, connecting hub nodes in such a way that the hub-level network remains connected with undirected connections (edges). The remaining $n-p$ spoke nodes will be allocated to different hub nodes from among the p hubs. The spoke level nodes form directed rotation services starting from the hub node, visiting all the spoke nodes allocated to it and returning to the hub node. There will be at least two spoke nodes allocated to every hub node unless practical real-life situations do not permit this, in which case the sole allocated spoke node is called an isolated spoke node and is spurred at the hub node. A runaway node is an upgraded spoke node chosen from among the spoke nodes allocated to a given hub in order to provide a second access to outside the rotation in addition to the one provided by the hub node. Every cycle will include one runaway node, which is connected to two other such nodes on different rotations. The runaway connections are undirected. A discount factor, $\alpha$, represents the economies of scale at the hub-level and a $\lambda$ counterpart $(\alpha \leq \lambda \leq 1$ as the runaway connections do not provide the full function of a hub edge and as a result, the factor of economies of scale are less advantageous for the runaways) applies to the runaway connections. The objective is to find the optimal structure minimizing the total costs associated to setting up facilities and transportation costs on such a structure.

\section{Mixed-integer linear PRogramming Formulations}

The variables of model follow: $x_{i j}=1(\forall i \neq j)$, if node $i$ is allocated to hub $j, 0$, otherwise; $h_{i}=1$ if $i$ is a hub, 0 , otherwise; $y_{i j}=1(\forall i \neq j)$, if there exists a spoke $\operatorname{arc}(i, j), 0$ otherwise; $b_{i j}=1(\forall i<j)$, if a hub edge $\{i, j\}$ is established between two hub nodes $i$ and $j, 0$ otherwise; $z_{i j}=1(\forall i<j)$, if there exists a runaway connection $\{i, j\}$ between $i$ and $j, 0$ otherwise; $g_{i}=1$, if $i$ is runaway node, 0 otherwise; $w_{i j k l}$ represents the fraction of flow from $i$ to $j$ routed via hub edge $(k, l) ; s_{i j k l}$ stands for the fraction of flow from $i$ to $j$ routed via spoke edge $(k, l)$ and $v_{i j k l}$, represents the fraction of flow from $i$ to $j$ routed via runaway connection $(k, l)$.

Additionally, for any subset $S \subset V, \delta^{+}(S)=\{a=(i, j) \mid i \in S, j \in V / S\}, \delta^{-}(S)=\{a=(j, i) \mid i \in S, j \in V / S\}$, $y\left(\delta(S)^{+}\right)=\sum_{i \in S, j \in V / S} y_{i j}$.

The $p$-Hub Location Problem with Runaway ( $p$ HLPwR) model can be stated as follows:

$$
\begin{aligned}
& \min \sum_{i=1}^{n} \sum_{\substack{j=1 \\
j \neq i}}^{n} \sum_{\substack{k=1 \\
k \neq j}}^{n} \sum_{\substack{l=1 \\
l \neq k \\
l \neq i}}^{n} w_{i j} c_{k l}\left(s_{i j k l}+\lambda v_{i j k l}+\alpha w_{i j k l}\right) \\
& \quad+\sum_{k=1}^{n} \sum_{\substack{l=1 \\
l>k}}^{n} I_{k l} b_{k l}+\sum_{k=1}^{n} \sum_{\substack{l=1 \\
l \neq k}}^{n} S_{k l} y_{k l}+\sum_{k=1}^{n} \sum_{\substack{l=1 \\
l>k}}^{n} T_{k l} z_{k l} \\
& \quad+\sum_{k=1}^{n} F_{k} h_{k}+\sum_{k=1}^{n} G_{k} g_{k} \\
& \text { s.t. } \sum_{j=1}^{n} h_{j}=p, \\
& \quad x_{i j} \leq h_{j}, \\
& \sum_{j=1, j \neq i}^{n} x_{i j}+h_{i}=1, \\
& g_{i}+h_{i} \leq 1 \\
& \sum_{j>i}^{n} z_{i j}+\sum_{j<i}^{n} z_{j i}=2 g_{i},
\end{aligned}
$$




$$
\begin{aligned}
& \sum_{i=1}^{n} \sum_{j>i}^{n} z_{i j} \leq p \\
& z_{i j} \leq g_{j} \\
& g_{i}+x_{i k}+g_{j}+x_{j k} \leq 3, \\
& \forall i \in V, j>i, \\
& \forall i, j, k \in V, k \neq j, k \neq i, j>i \text {, } \\
& y_{i j}+x_{i k} \leq 1+x_{j k} \text {, } \\
& y_{i j}+h_{i} \leq 1+x_{j i} \text {, } \\
& y_{i j}+x_{i j} \leq 1+h_{j} \text {, } \\
& y_{i j}+y_{j i}+x_{k i}+x_{k j} \leq 3-h_{i}-h_{j} \text {, } \\
& \sum_{j=1, j \neq i}^{n} y_{i j}=1 \text {, } \\
& \sum_{j=1, j \neq i}^{n} y_{j i}=1, \\
& b_{i j} \leq h_{i}, \\
& b_{i j} \leq h_{j} \text {, } \\
& \sum_{l=1, l \neq i}^{n} w_{i j i l}+\sum_{l=1, l \neq i}^{n} v_{i j i l}+\sum_{l=1, l \neq i}^{n} s_{i j i l}=1, \\
& \sum_{l=1, l \neq j}^{n} w_{i j l j}+\sum_{l=1, l \neq j}^{n} v_{i j l j}+\sum_{l=1, l \neq j}^{n} s_{i j l j}=1, \\
& \sum_{\substack{l=1 \\
l \neq i, l \neq k}}^{n} w_{i j k l}+\sum_{\substack{l=1, l \neq i, l \neq k}}^{n} v_{i j k l}+\sum_{\substack{l=1, l \neq i \\
l \neq k}}^{n} s_{i j k l} \\
& =\sum_{\substack{l=1, l \neq j, l \neq k}}^{n} w_{i j l k}+\sum_{\substack{l=1, l \neq j, l \neq k}}^{n} v_{i j l k}+\sum_{\substack{l=1, l \neq j, l \neq k}}^{n} s_{i j l k}, \\
& \forall i, j, k \in V, k \neq i, k \neq j, j \neq i, \\
& \forall i, j, k \in V, k \neq j, j \neq i, \\
& \forall i, j, k \in V, k \neq i, j \neq i, \\
& \forall i, j, k \in V, k \neq j, k \neq i, j>i \text {, } \\
& \forall i \in V \text {, } \\
& \forall i \in V \text {, } \\
& \forall i, j \in V, j>i \text {, } \\
& \forall i, j \in V, j>i \text {, } \\
& \forall i, j \in V, j \neq i \text {, } \\
& \forall i, j \in V, j \neq i, \\
& s_{i j k l} \leq y_{k l} \text {, } \\
& v_{i j k l}+v_{i j l k} \leq z_{k l}, \\
& w_{i j k l}+w_{i j l k} \leq b_{k l}, \\
& y\left(\delta^{+}(S)\right) \geq \sum_{j \in V / S} x_{i j}, \\
& y\left(\delta^{-}(S)\right) \geq \sum_{j \in V / S} x_{i j}, \\
& h_{i}, g_{i}, x_{i j}, y_{i j}, z_{i k}, b_{i k} \in\{0,1\}, \\
& w_{i j k l}, v_{i j k l}, s_{i j k l} \in(0,1) \text {, }
\end{aligned}
$$

The objective function minimizes the transportation costs and the setup costs for hubs, runaways, hub edges, runaway connections and spoke arcs. Constraints (3.2) ensure that the number of hubs is equal to $p$. Constraints (3.3) guarantee that a node $i$ can be allocated to node $j$, only if node $j$ is a hub. Constraints (3.4) ensure that a node $i$ is either a hub node or is allocated to only one hub. Constraints (3.5) assure that a node $i$ cannot be a hub and a runaway node at the same time. Constraints (3.6) ensure that a runaway node must be adjacent to 


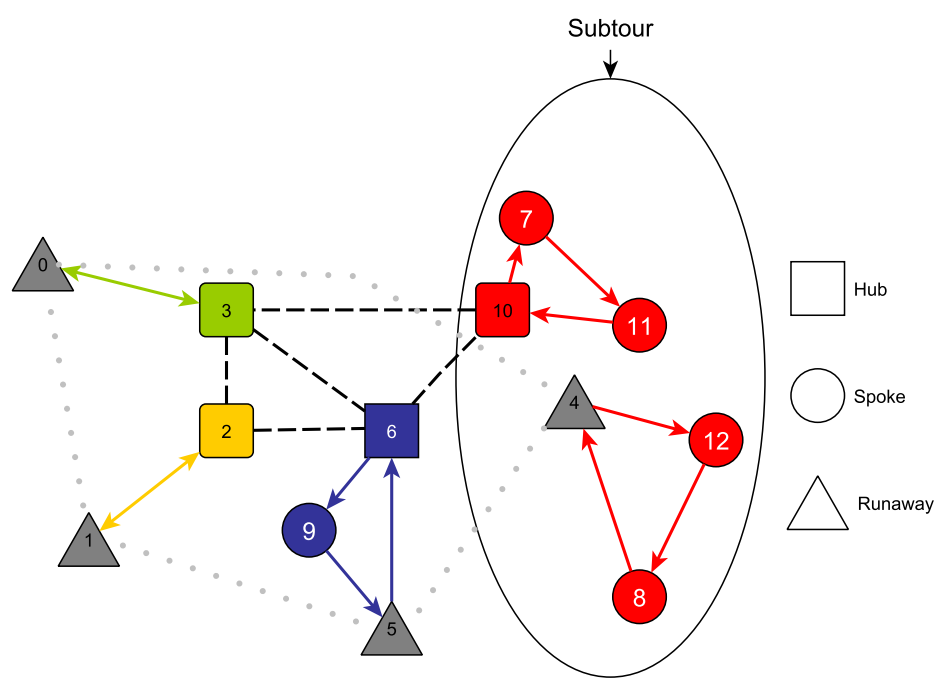

Figure 4. Subtour example $(n=13, p=4)$.

two runaway connections. Constraints (3.7) assure that the number of runaway connections is at most equal to $p$. Constraints (3.8) ensure that if there is a runaway connection $(i, j)$, then $j$ is a runaway node. Constraints (3.9) ensure that in each cycle there is at most one runaway node. Constraints (3.10)-(3.12) guarantee that a spoke arc links two spokes of the same hub (cycle). Constraints (3.13) are aggregations of the two constraints $y_{i j}+y_{j i} \leq 1$ and $x_{k i}+x_{k j} \leq 2-h_{i}-h_{j}$. The former makes sure that two spoke arcs in opposite directions do not exist and the later guarantees that one spoke node cannot be allocated to two hub nodes at the same time.

Constraints (3.14) ensure that a spoke $i$ has exactly one outgoing spoke arc. Constraints (3.15) ensure that a spoke i has exactly one incoming spoke arc. Constraints (3.16) and (3.17) ensure that if the hub edge $b_{i j}$ exists then the nodes $i$ and $j$ are hubs. Constraints (3.18) assure an origin-destination flow $i-j$ leaves $i$ via a spoke, a hub or a runaway connection. Constraints (3.19) assure that an origin-destination flow $i-j$ arrives to its destination $j$ via a spoke, a hub or a runaway connection. Constraints (3.20) assure that flow conservation holds at every intermediate node visited along a origin-destination path for flow $i-j$.

Constraints (3.21)-(3.23) guarantee that a flow between two nodes $i$ and $j$ will traverse the link $k-l$ (spoke arc, runaway connection or hub edge) if compatible link exists.

Constraints (3.24) and (3.25) ensure that $\forall i \in S \subset V$ if $i$ is a spoke node allocated to a hub in $V / S$, there is at least one arc going out of (entering into) $S$. For any given subset $S \subset V$ and any $i \in S$, in the absence of any arc $(i, j)$ with the tail in $S$ and the head in the complementary set, any path from $i$ to the hub serving $i$ entirely lies within $S$. Thus, there exists no $j$ outside $S$, which serves $i$ for any choice of $j \in V / S$. On the contrary, if $i$ is served by a hub in $V / S$ there is a unique path cutting $S$ (at least once) towards the hub serving $i$.

Constraints (3.24) and (3.25) ensure that situations similar to Figure 4 will not occur.

\section{HyPER-HEURISTIC AND VNS APPROACHES FOR PHLPwR}

The complexity of our problem and its computational intractability make it impractical to solve realistic size instances. We therefore propose a hyper-heuristic and a VNS approaches aiming to find high quality solutions for larger instances in a reasonable amount of time. 


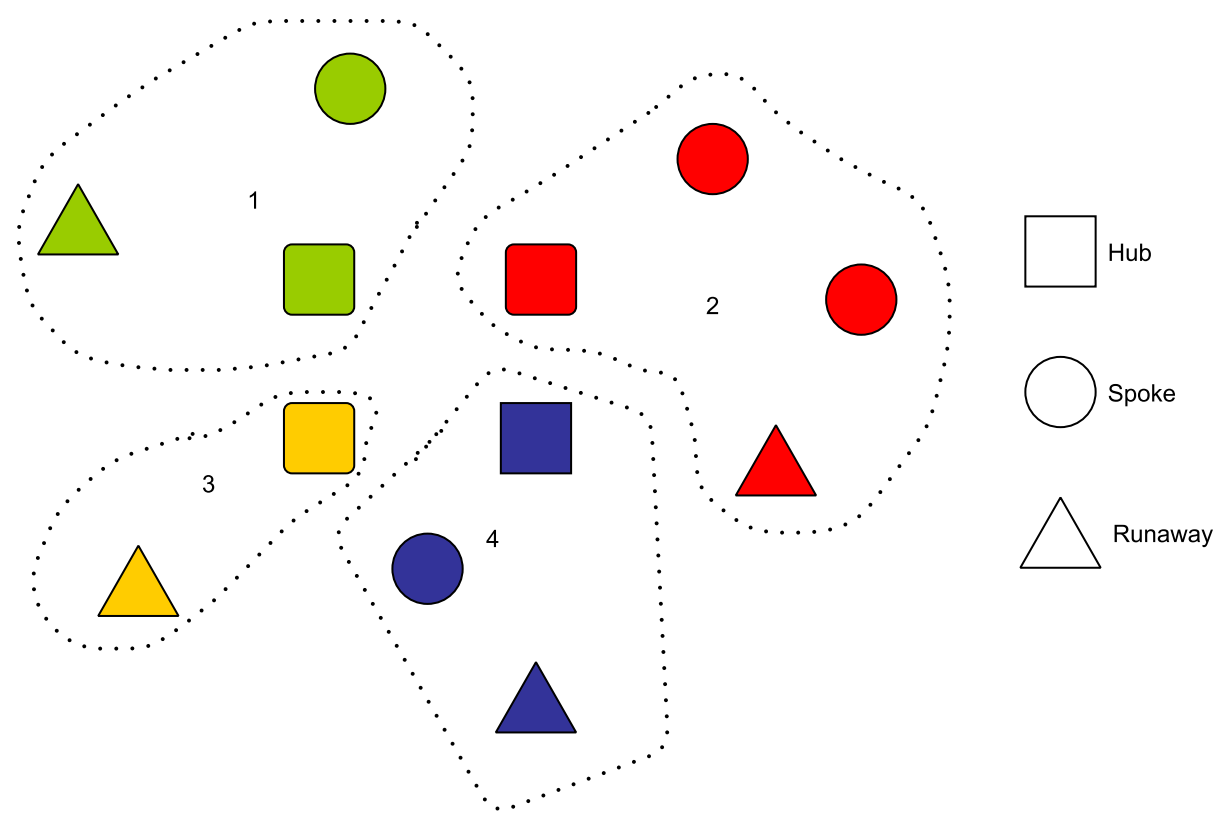

FiguRE 5. pHLPwR without the network structure ( $p=4$ clusters).

\subsection{Hyper-heuristic approach}

The term hyper-heuristic was first used in 1997 to describe a protocol that combines several artificial intelligence methods in the context of automated theorem proving [17]. It was then employed in combinatorial optimization [13] as heuristics to choose heuristics. In this viewpoint, a hyper-heuristic is a high-level approach that solves hard computational search problems, given a particular problem instance and a number of low-level heuristics. It selects and applies the most suitable low-level heuristic at every decisional milestone. The main ingredients of our hyper-heuristic are elaborated in the sequel.

\subsubsection{Initial solution}

An initial solution, or more importantly a good one, empirically speaking, contributes significantly in the success of many methods (even exact ones) and a hyper-heuristic is of no exception. In the case of this problem, we have carried out an extensive preliminary computational experiments that confirmed again the importance of a good quality initial solution.

A solution to this problem is characterized by (1) location of hub nodes, (2) runaway nodes location, (3) spokes allocation, (4) hub-level, runaway-level structures, and the spoke-level network (i.e. the cycles).

One can start with a clustering problem. Figure 5 shows that for $p=4$, we obtain 4 clusters, and every cluster contains a single runaway (of course if it does not only serving a single isolated node, in which case there will be no runaway) node and one and only one hub node. One of the most popular unsupervised clustering methods is the well-known $k$-means method [28], due to its ease of implementation and efficiency. Interested readers are referred to some of the related recent contributions including Pérez-Ortega et al. [38], Huang et al. [24], Mourelo Ferrandez et al. [34], Todosijević et al. [45], Yahyaei et al. [47] and O'Kelly [36].

The main steps of our pHLPwR implemented $k$-means are described in the Algorithm 1 ( $k=p$ the number of hubs). First, we initialize the centroids [23]. Then, we construct the clusters. Finally, we locate the hub - as the nearest node to the centroid - and a runaway node for every cluster - chosen to be the farthest node to the centroid. The reason is that one would expect this node to be sufficiently far from the surrounding area of the disrupted hub node. 


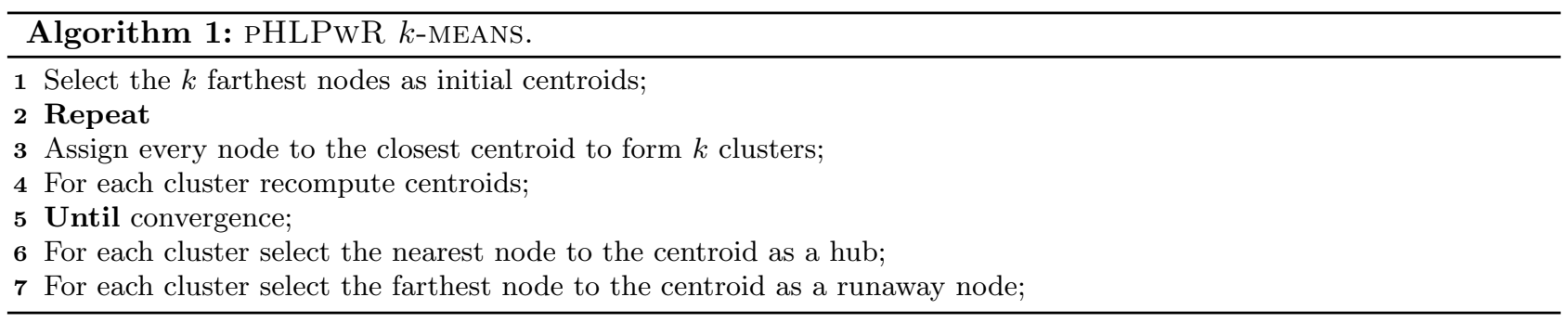

Once Algorithm 1 terminates, we inter-connect all the hubs (complete graph) and construct the cycles for each cluster using a greedy constructive heuristic choosing the cheapest edge to add at every step. Our extensive preliminary computational experiments has revealed that this method is effective and provides quality initial solution for our hyper-heuristic.

\subsubsection{Selection method}

The selection procedure is the main component of a heuristic-selection hyper-heuristic. A good selection procedure leads to a better decisions when choosing the low-level heuristic to apply and therefore finding good solutions becomes more likely. The selection approach used in this study belongs to the class of reinforcement learning methods (see [4]). A reinforcement learning approach is based on a scoring system. In this work we use an on-line learning approach, each time a low-level heuristic is called, its weight value is updated dynamically; rewarded, if the low-level improves the solution or penalized, if it worsen the solution.

We define the weight variable as in the following [15]:

$$
w_{i}=\left(\sum_{1}^{N_{i}}\left(f^{\text {in }}-f^{\text {out }}\right)\right) / N_{i} .
$$

The weight $\left(w_{i}\right)$ of a low-level heuristic $i$ is equal to the negative sum (minimization case) of the difference in objective function values after applying the low-level heuristic $i$ divided by the total number of times that it has been called during the search where $f^{\text {in }}$ is the current objective value, $f^{\text {out }}$ is the objective value after calling the low-level heuristic $i$ and $N_{i}$ is the number of times the low-level heuristic $i$ was used. This weight gives an on-average effectiveness of a low-level heuristic. In other terms, a low-level heuristic with a higher value would probably return better solutions.

The low-level heuristics weights are initialized according to their performances on the initial solution. In fact, after creating the initial solution, we let every heuristic try to improve it until meeting a stopping criteria (the number of non-improving solutions). The equation (4.1) is used to calculate the initial weights.

It must be noted that we have also carried out extensive computational results to see if initializing with random weights would be any better as more exploration should be introduced. However, the conclusion out of numerous observations was that no clear pattern of impact on the performance of method - neither clearly improving nor really deteriorating - could be found. Therefore, we have decided to not include them in this study.

The selection procedure (Algorithm 2) chooses the low-level heuristic $i$ with the highest weight and $n c_{i}$ less or equal to the parameter $R_{l}$ ( $R_{l}$ is the maximum authorized consecutive calls) and increments the number of consecutive calls of the low-level heuristic, $i$, i.e. $n c_{i}$ (see line 11)). If $R_{l}=1$, then the selection procedure will select a sequence of low-level heuristics from the best to the worst.

\subsubsection{Low-level heuristics}

In the following we define the neighborhood structures and the set of low-level heuristics used in our hyperheuristic. We will also briefly explain how each of them performs: 


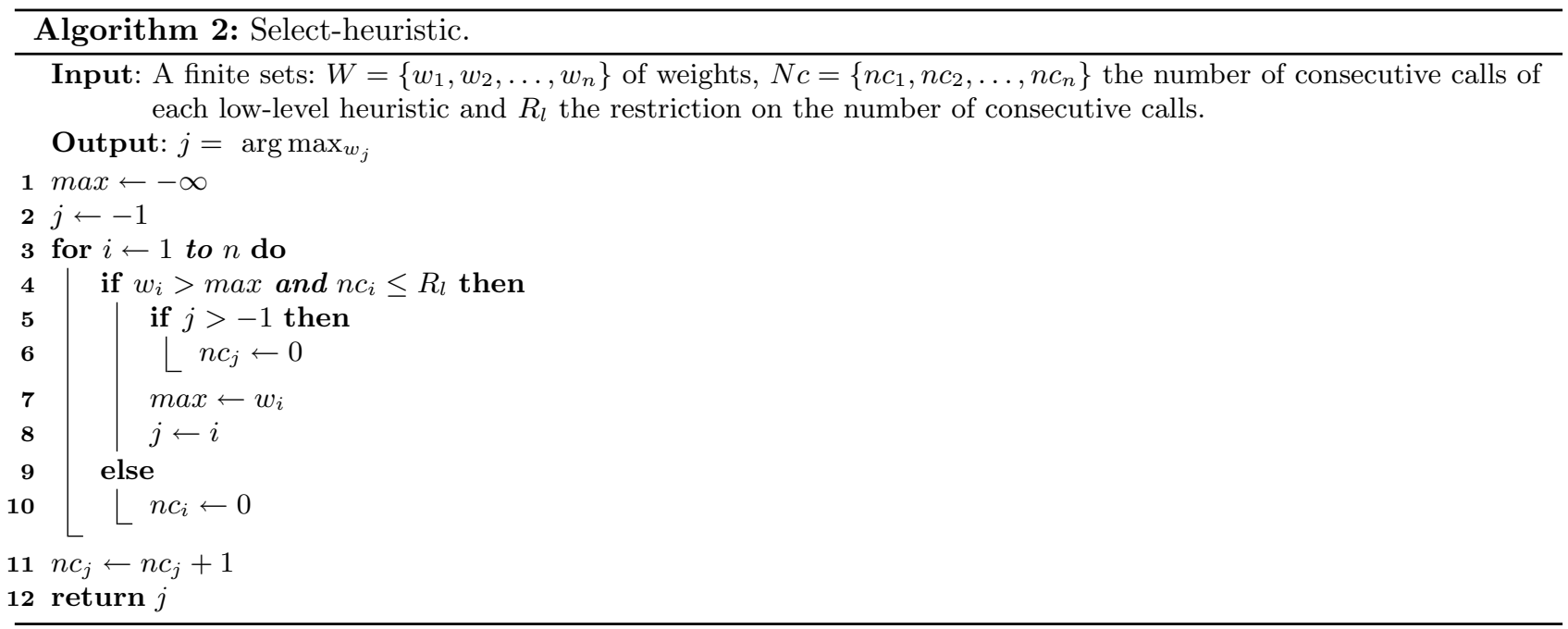

Network low-level heuristics. These heuristics tend to improve the solution without altering the membership of nodes to the clusters.

LINK-HUB: this low-level heuristic adds or deletes a hub edge in the hub-level network for each pair of hubs. While adding a hub edge between two hub nodes, if not already available, does not harm the feasibility, a deletion can cause infeasibility and disconnectedness in the hub-level network in which case the solution is discarded.

LINK-RUNAWAY: add and/or delete a runaway connection to get a new runaway-level network. The number of runaway connections incident to each runaway node must not exceed 2.

NEW-CYCLE-LINKS: for the cycle attached to the hub node $k$ :

- Destroy the spoke-level network by removing all the spoke arcs of the cycle $k$.

- choose a node $i$ as a start and use the nearest neighbor heuristic to create a solution for the traveling salesman problem (TSP) of the nodes in the cluster and complete the tour.

Distribution low-level heuristics. These local searches try to improve every cycle without changing the number of nodes allocated to it (swapping between nodes).

SWAP-CYCLE-NODES: for each spoke node $i$ allocated to cycle $k$ :

- Find the nearest node $j$ to the node $i$ ( $j$ allocated to cycle $k$ ).

- Swap the positions of the spokes $i$ and $j$ on the spoke-level network.

SWAP-SPOKE-RUNAWAY: for every spoke node, we swap this node with its corresponding runaway node such that the spoke becomes runaway and the runaway becomes a spoke.

SWAP-SPOKE-HUB: we swap every spoke with its corresponding hub such that the spoke node becomes the hub and the hub node becomes a spoke node.

SWAP-DIFFERENT-CYCLE: for each spoke node $i$ allocated to hub $k$ :

- Find the nearest hub $l$ to the hub $k$.

- Find a node among the spokes allocated to hub $l$, say spoke $j$, which is the nearest node to the spoke $i$.

- Swap the spoke $i$ with the spoke $j$ so that the spoke $i$ takes the position of spoke $j$ in the cycle attached to the hub node $l$ and the other way around.

Structure low-level heuristics. The objective is to find the best cluster composition (moving nodes among clusters).

MOVE-SPOKE-NEAREST-CYCLE: for each spoke node $i$ allocated to hub node $k$ : 
- Find the nearest hub $l$ to the hub $k$.

- Find a node among spokes allocated to hub $l$, say spoke $j$, which is the nearest node to the spoke $i$.

- Remove spoke $i$ from cycle attached to the hub node $k$.

- Add the spoke node $i$ in cycle attached to the hub node $l$ before/after the spoke $j$ in the spoke-level network.

MOVE-SPOKE-NEAREST-NODE: this low-level heuristic finds for a given spoke node $i$ allocated to a hub node $k$, the nearest spoke node $j$ in the whole network (not only in the nearest cycle to $k$ as seen in the precedent low-level heuristic). After finding the spoke node $j$ with its corresponding hub node $l$ :

- Removes the spoke node $i$ from cycle attached to the hub node $k$.

- Add the spoke node $i$ to cycle attached to the hub node $l$ before/after the spoke node $j$ in the spoke-level network.

\subsubsection{Hyper-heuristic procedure}

While in meta-heuristics the search space is usually the space of solutions and the main goal is to find the best solution, in a hyper-heuristic, the search space is the space of low-level heuristics and therefore the objective is to find, at each step, the best low-level heuristic to apply in order to obtain the best feasible solution. Before explaining our algorithm process we give two definitions to ease understanding.

Move acceptance strategy. The solution obtained at the end of an invocation of a low-level heuristic can be accepted or rejected according to the move acceptance strategy being used. In the case of our algorithm, we use a random walk acceptance criteria, where a solution is accepted regardless of its quality. This strategy adds some noise to the search path, which avoids premature convergence to a local optimum.

Perturbation heuristic. The role of this heuristic is to prevent getting stuck in a local optima by giving distant solutions. In other terms, the objective is to push towards more exploration to search possibly unexplored areas in the search space rather than immediately improving the current solution.

- DESTROY : it partially destroys a solution. For each hub with more than $n / p$ allocated spoke nodes, the cycle is disconnected by removing all the corresponding spoke edges.

- REALLOCATE : every disconnected spoke is reallocated to the nearest hub among the current hub nodes.

- CONSTRUCT : for each hub and its corresponding spoke nodes, spoke edges are added step by step using the nearest neighbor heuristic in order to reconstruct a new cycle.

In order to increase the likelihood of escaping from the local optima and finding a promising solution search space area, the perturbation heuristic accepts as input, the best-found solution rather than the latest visited node.

Our hyper-heuristic starts with the calculation of the initial weights according to the definition seen in Section 4.1.2. The low-level heuristic with the highest weight is designated. Subsequently, the best solution found so far and its objective value are recorded in $x^{\prime}$ and min, respectively. If $x^{\prime}$ is the best solution found during the process, it will be stored in $x^{*}$ and its objective function value in top. At every call to a low-level heuristic $i$, its weight $w_{i}$ is updated according to (4.1). The perturbation heuristic is called $d$ times before reaching the stopping criteria and takes, $x^{*}$, as an entry. We set our termination criteria as the number of consecutive non-improving moves, $N_{\text {limit }}$, or the time limit, $T_{\text {limit }}$. A tabu list $T_{l}$ is also added. The tabu list is a short-term memory that holds the recently visited solutions to prevent the same solutions to be reconsidered in the near future and a possible cycling in the same search space area. The tabu tenure that provides good results often grows with the size of the problem, that is why we have chosen $n$ as the tabu tenure. In other terms, a solution remains tabu for $n$ iterations then becomes non-tabu.

One can summarize the algorithm process in seven main steps (see Algorithm 3). (1) the low-level heuristics weights are initialized (line 2), (2) After each $N_{\text {limit }} / d$ non-improving moves (line 7 ) the perturbation heuristic is called on the best-found solution (line 8), otherwise the low-level heuristic with the best performance so far, is applied on the current solution (line 10), (3) the neighborhood is explored for the best solution, which is not in the tabu list (line 16). The solution is then considered as current incumbent solution $x^{\prime}$, even if it is a 
TABLE 2. The parameters used in Algorithm 3.

\begin{tabular}{ll}
\hline \hline Parameter & Definition \\
\hline$d$ & The number of perturbation heuristic calls to escape from a local optimum \\
$T_{l}$ & The tabu list \\
nbr_iter & The number of successive worsening moves \\
$T_{\text {limit }}$ & The time limit \\
$N_{\text {limit }}$ & The number of worsening moves tolerated \\
sols & An array of solutions \\
$x^{\prime}$ & The current solution (incumbent) \\
$x *$ & The best solution found \\
\hline
\end{tabular}

non-improving move (line 18), (4) the incumbent solution found $x^{\prime}$ is added to the tabu list $T_{l}$ and if the size of $T_{l}$ exceeds $n$, the oldest solution is removed in a first-in, first-out (FIFO) scheme (line 20). (5) the weight of the low-level heuristic is updated (line 21), (6) if the current solution improves over the best-found solution, we update the best-found solution, $x^{*},(7)$ steps $2-7$ are iterated until the termination criteria is met (line 6).

The parameters of our algorithm are presented in Table 2.

\subsection{Variable neighborhood search approach}

Our hyper-heuristic is a rather comprehensive and complete framework and our goal is not to present a second metaheuristic approach as a state-of-the-art method for this problem. Our aim is to show how a combination of components of our hyper-heuristic would perform in the absence of hyper-heuristic settings. Therefore, a variable neighborhood search (VNS) [31] scheme perhaps makes this comparison more reasonable and can be referred to as a comparable counterpart to our hyper-heuristic.

Let $\mathcal{N}=\left\{N_{1}, \ldots, N_{k_{\max }}\right\}$ be a set of neighborhood structures. The Algorithm 4 iterates over four main steps until the stopping criteria is met (line 6): (1) calls the Shaking heuristic with neighborhood structure $N_{i}$ (line 8), (2) performs the LocalSearch procedure (line 9), (3) updates the current solution in $x^{*}$ (line 12) if an improvement is observed (line 11), (4) examines the subsequent neighborhood $\left(N_{i+1}\right)$ if no improvements reported (line 17).

The aim of the Shaking procedure is to change and explore other interesting search space areas in the neighborhood $N_{k}$ and to avoid the local optimum. On the other hand, the role of the LocalSearch procedure is exploring and discovering the descent direction within neighborhood $N_{k}$ to reach a local optimum. Figure 6 summarizes the VNS scheme.

For a fair comparison between the two algorithms, we have used the low-level heuristics of the hyper-heuristic to construct the Shaking neighborhoods and the LocalSearch procedure. We considered the perturbation operators as the Shaking neighborhoods, and the other low-level heuristics as the LocalSearch procedure (see Sect. 4.1.3). In doing so, every solution obtained with the hyper-heuristic can be obtained by the VNS algorithm as well. Our implemented version of VNS has the same stopping criteria as the hyper-heuristic (time limit $T_{\text {limit }}$ or non-improving moves $N_{\text {limit }}$ ).

Interested readers on the VNS applications in HLPs are referred to the recent works in the literature Dai et al. [14], Serper and Alumur Alev [43], Rahmaniani et al. [39], Jarboui et al. [25] and Pérez et al. [37].

\subsection{Cutting planes}

As the proposed model is not a compact one, valid inequalities (3.24) and (3.25) need to be separated on the fly and upon violation. 

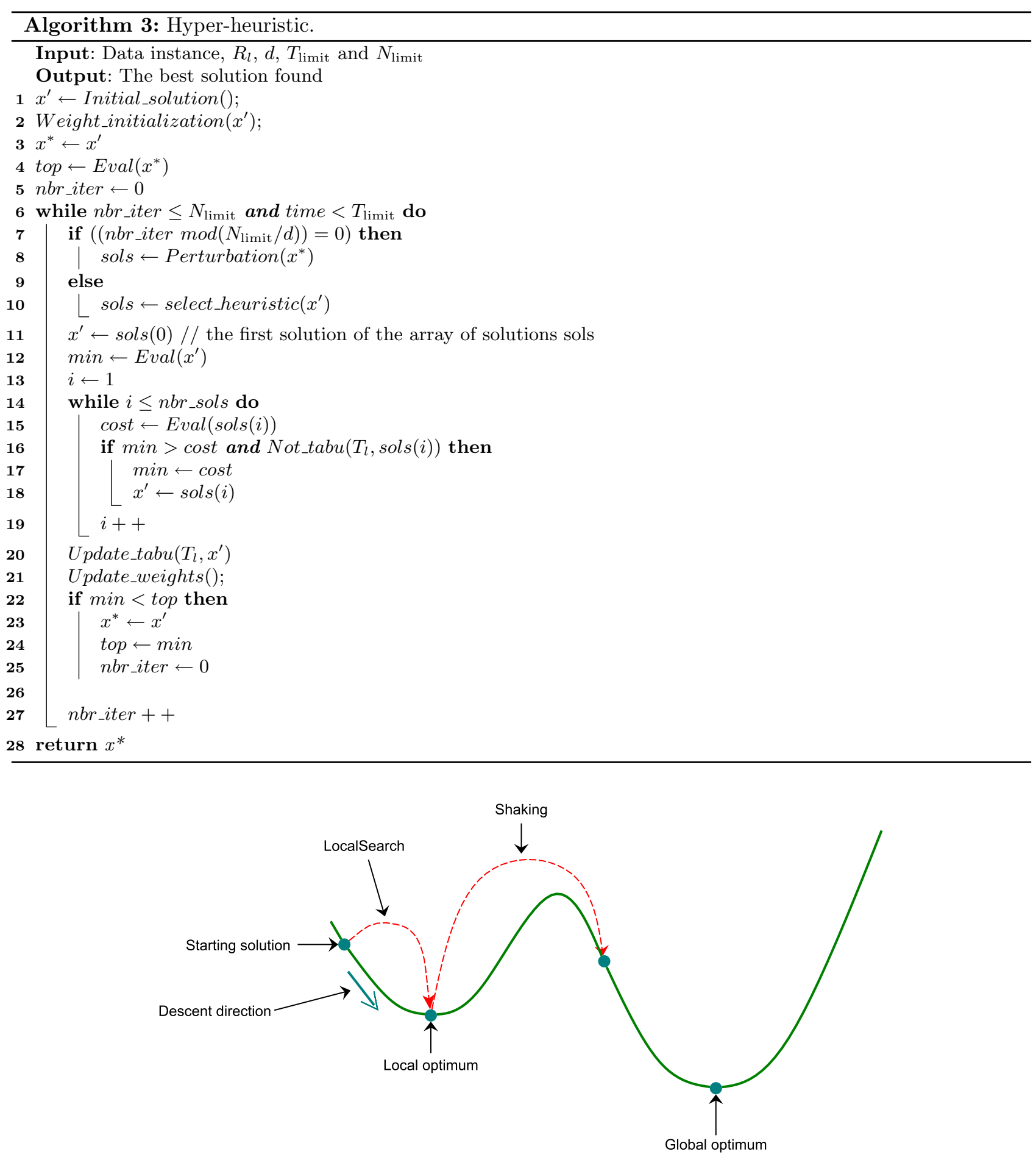

FiguRE 6. VNS basic scheme. 


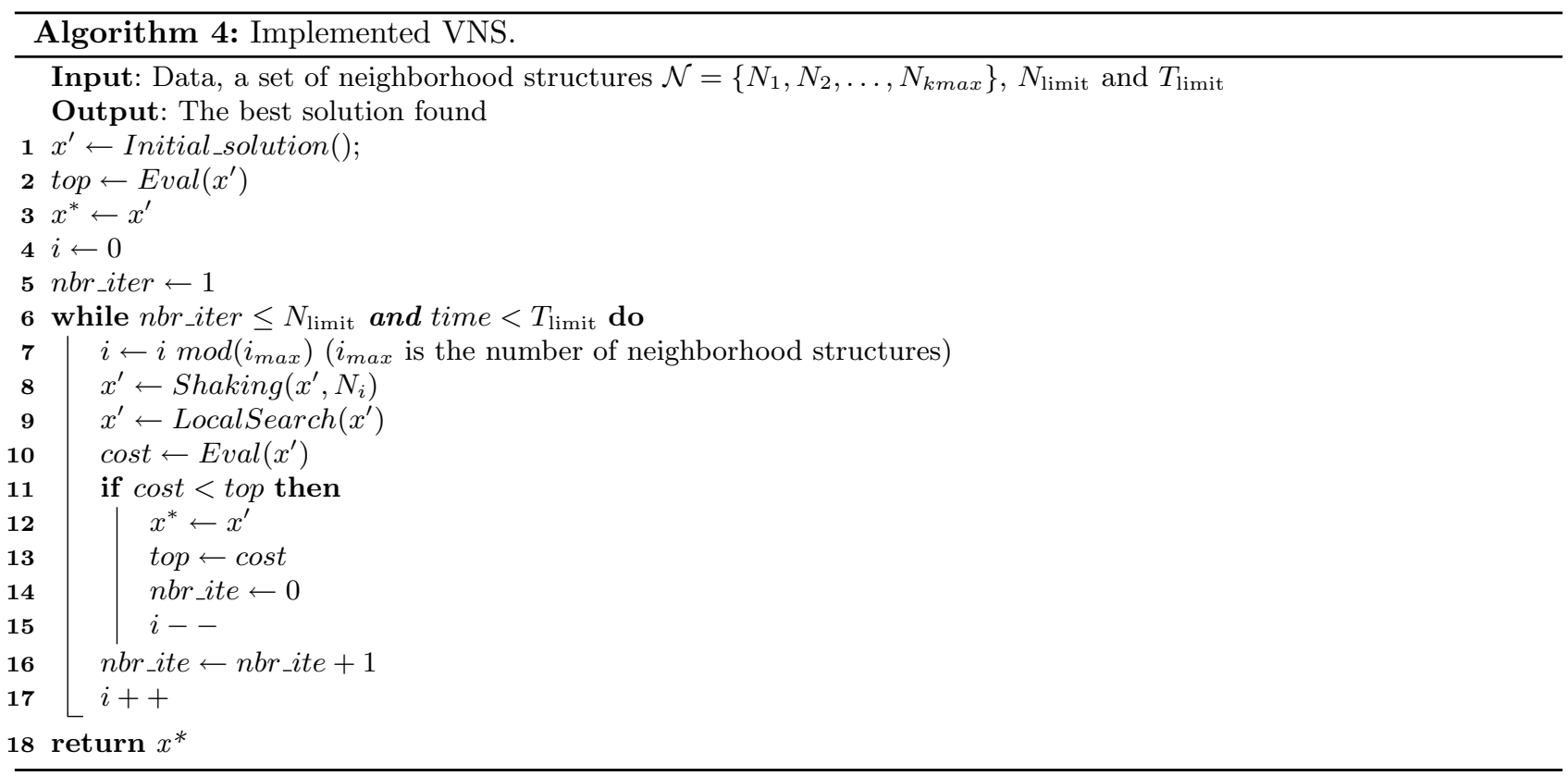

Separation of valid inequalities (3.24) and (3.25). Let $\mathrm{s}$ be a dummy node, $V$ be the set of nodes visited and $G^{\prime}(V \cup\{s\}, A)$ a directed graph. For each $i$ establish an arc from $s$ to every $j$ with capacity $\bar{x}_{i j}$, if $\bar{x}_{i j}>0$. Then, add an $\operatorname{arc}(i, j)$ with capacity $\bar{y}_{i j}$ for $\bar{y}_{i j}>0$. Now, if we push one unit of flow from $s$ destined to a node $i$, then $i$ must receive a volume of flow equivalent to $\sum_{j} \bar{x}_{i j}$. A set $S \subset V$ where $i \in S, s \notin S$ defines a cut of $\delta^{+}(S)\left(\delta^{-}(S)\right)$ if the cut capacity is less than $\sum_{j} \bar{x}_{i j} . S$ will deliver valid inequalities of (3.24) (3.25).

\section{Computational Results}

Due to the confidentiality, we are only able to report our results on the instances generated based on the well-known Australian Post (AP) dataset [19]. The fixed costs are generated based on the distance and flow to get realistic costs as in the following (see [20]):

- Hub nodes: $F_{k}=\left(\sum_{i=1}^{n} \sum_{j=1, j \neq i}^{n} w_{i j} / \max _{i \neq k} d_{i k}\right) \times 10 \mathrm{e}^{8}$, where $\max _{i \neq k} d_{i k}$ is the distance between the most remote location to $k$. If this distance is a large number, the node $k$ is far from the remaining nodes and therefore $F_{k}$ is less expensive.

- runaway nodes: $G_{k}=\frac{1}{2}\left(\sum_{i=1}^{n} \sum_{j=1, j \neq i}^{n} w_{i j} / \max _{i \neq k} d_{i k}\right) \times 10 \mathrm{e}^{8} \cdot G_{k}$ is calculated in the same way as $F_{k}$ but slightly cheaper than hub nodes.

- Hub edges: $I_{k l}=\left(\frac{w_{k l} / d_{k l}}{\max _{i, j \neq i} w_{i j} / d_{i j}}\right) \times 10 \mathrm{e}^{7}$. This function is based on the distance and flow to get the importance of each edge and its cost. A high cost $I_{k l}$ means that the edge $k-l$ transits a high flow $w_{k l}$ and(or) $k$ is close to $l$.

- runaway edge: $T_{k l}=\frac{1}{2}\left(\frac{w_{k l} / d_{k l}}{\max _{i, j \neq i} w_{i j} / d_{i j}}\right) \times 10 \mathrm{e}^{7} . T_{k l}$ is calculated with respect to $I_{k l}$ (a runaway edge is less expensive than a hub edge).

- Spoke edge: $S_{k l}=\frac{1}{4}\left(\frac{w_{k l} / d_{k l}}{\max _{i, j \neq i} w_{i j} / d_{i j}}\right) \times 10 \mathrm{e}^{7} \cdot S_{k l}$ is calculated with respect to $I_{k l}$ (a spoke edge is less expensive than a hub and runaway edge).

The costs were generated according to the importance of each node and edge. The more a node or a edge is important, the higher the cost is. 
The name of each instance is referred as nwwpxx_yy_zz where $w w$ is the number of nodes, $x x$ is the number of hubs, $y y$ is the hub-level discount factor $\alpha$ and $z z$ is the runaway connection discount factor $\lambda(\alpha \leq \lambda \leq 1)$. Three combinations have been taken into account, namely, $(\alpha, \lambda) \in\{(0.6,0.8),(0.8 .0 .9),(0.9,1)\}$ to avoid too many similarities and have a better approximation of real-life cases. $\lambda=1$ does not distinguish between the spoke connection and a runaway connection.

The algorithms were implemented in C++ and CPLEX 12.8.0 was used as MIP solver for solving our mathematical model for different instances. The experiments were performed on an Intel $2.10 \mathrm{GHz}$ core i7 CPU with 8 GB RAM running on Windows 10.

In the tables below, MIP-HLPwR refers to the exact branch-and-cut method (solving with CPLEX and separating from among the exponential number of constraints), H-HLPwR stands for the hyper-heuristic method and VNS-HLPwR refers to the prescribed VNS method.

\subsection{Branch-and-cut approach}

In this section, we examine only the computational behavior of the MIP model when CPLEX is being used as a modern (equipped with callbacks) MIP solver and the exponential constraints are separated via a IloUserCutCallback (see Sect. 4.3). The time limit was set to $86400 \mathrm{~s}$ (i.e. 1 day) and the tolerance $\epsilon$ is equal to 1e-6. Constraints (3.24) and (3.25) were separated at every integer node (feasible solution).

The results are reported in Table 3 where "Obj. Val." is the objective value of the best solution found. "NSols" represent the number of feasible solutions found; "E.T." stands for the execution time; the column "GAP" reports the relative gap returned by CPLEX upon termination; "Nnodes" gives the number of nodes processed by CPLEX; "Status" reports the status of CPLEX when termination criteria is met; "best obj." reports the best lower bound found by CPLEX and "Ncuts" represents the number of separated sub-tour elimination cuts.

Generally speaking, the objective value increases with the size of the instance and for the same instance size, $n$, the smaller $p$ construct an upper envelop on the objective function of instances with a higher $p$ (see Fig. 7b).

Figure 7a shows the relation between $\alpha$ and $\lambda$ with the computational difficulties of the problem. For the same instance size, the smaller $\alpha$ and $\lambda$ are, the higher is the computational time needed to prove optimality. Indeed, the smallest execution times recorded are for $\alpha=0.9, \lambda=1$ (see Tab. 3) where runaway connections offers no advantage over the spoke edges. As we only had provably optimal solutions for up to size $n=15$, Figure 7a depicts only up to this size.

Most of the instances with less than 20 nodes were solved to optimality in less than $24 \mathrm{~h}$. However, for $15-25$ nodes, CPLEX often finds a feasible solution but needs more time to prove optimality. CPLEX encounters serious difficulties when dealing with instances of more than 20 nodes - even delivering a feasible solution remains a challenge.

We observe that the number of cuts added correlates to the number of hub nodes $p$. The lower the $p$ is, compared to the number of nodes $n$, the higher is the number of cuts to be separated. This sounds reasonable since the smaller $p / n$ is, the more nodes will sit on every cycle and as such, we may need more sub-tours to be eliminated. For $n=15, p=7$, no cuts are added because every cycle has at most three nodes and as such, we may not obtain sub-tours.

It must be noted that the number of sub-tour elimination separated remains reasonable.

\subsection{Hyper-heuristic vs VNS}

The results of the hyper-heuristic and VNS approaches are depicted in Table 4 for small size instances and Table 5 for medium and large size instances. Again, "Obj. Val." is the objective value of the best solution found. "NSols" represent the number of distinct feasible solutions encountered along the search, "E.T." stands for the execution time, the column "GAP" in Table 4 reports the relative GAP between the best-known solution of the hyper-heuristic (respectively best-known solution of VNS) and the best solution found by CPLEX. In Table 5 
TABLE 3. Computational results of MIP-HLPwR.

\begin{tabular}{|c|c|c|c|c|c|c|c|c|}
\hline Instance & Obj. Val. & NSols. & E.T. (s) & GAP $(\%)$ & Nnodes & Status & Best Obj. & NCuts \\
\hline n10p3_0.6_0.8 & 77593660.780 & 10 & 659.868 & 0.000 & 3558 & Optimal & 77593660.780 & 66 \\
\hline n10p3_0.8_0.9 & 82365794.641 & 7 & 725.919 & 0.000 & 3826 & Optimal & 82365794.641 & 83 \\
\hline n10p3_0.9_1 & 85258929.966 & 14 & 557.875 & 0.000 & 3560 & Optimal & 85258929.966 & 87 \\
\hline n11p3_0.6_0.8 & 20358494.269 & 24 & 8293.729 & 0.000 & 16369 & Optimal & 20358494.269 & 125 \\
\hline n11p3_0.8_0.9 & 21375615.575 & 11 & 6526.427 & 0.000 & 13438 & Optimal & 21375615.575 & 98 \\
\hline n11p3_0.9_1 & 22051911.670 & 18 & 6646.141 & 0.000 & 15101 & Optimal & 22051911.670 & 94 \\
\hline n11p4_0.6_0.8 & 17597082.029 & 10 & 772.829 & 0.000 & 2633 & Optimal & 17597082.029 & 34 \\
\hline n11p4_0.8_0.9 & 19128595.920 & 14 & 1049.542 & 0.000 & 3929 & Optimal & 19128595.920 & 29 \\
\hline n11p4_0.9_1 & 19835503.663 & 10 & 562.119 & 0.000 & 2391 & Optimal & 19835503.663 & 26 \\
\hline n12p4_0.6_0.8 & 20492068.237 & 14 & 10546.571 & 0.000 & 18470 & Optimal & 20492068.237 & 87 \\
\hline n12p4_0.8_0.9 & 21780501.640 & 10 & 6276.293 & 0.000 & 11316 & Optimal & 21780501.640 & 55 \\
\hline n12p4_0.9_1 & 22833868.689 & 10 & 7252.798 & 0.000 & 13666 & Optimal & 22833868.689 & 61 \\
\hline n13p5_0.6_0.8 & 22572799.697 & 15 & 13185.484 & 0.000 & 16054 & Optimal & 22572799.697 & 53 \\
\hline n13p5_0.8_0.9 & 24523121.813 & 12 & 10762.899 & 0.000 & 14012 & Optimal & 24523121.813 & 42 \\
\hline n13p5_0.9_1 & 25878687.968 & 8 & 9460.868 & 0.000 & 14012 & Optimal & 25878687.968 & 64 \\
\hline n14p5_0.6_0.8 & 39620089.247 & 16 & 86407.689 & 3.151 & 33465 & Feasible & 38371289.728 & 79 \\
\hline n14p5_0.8_0.9 & 43344082.949 & 17 & 86410.295 & 3.401 & 32961 & Feasible & 41869544.721 & 108 \\
\hline n14p5_0.9_1 & 45209210.686 & 11 & 79831.093 & 0.000 & 47822 & Optimal & 45209210.686 & 76 \\
\hline n14p6_0.6_0.8 & 36633192.377 & 7 & 10972.735 & 0.000 & 8570 & Optimal & 36633192.377 & 20 \\
\hline n14p6_0.8_0.9 & 40507279.264 & 11 & 10087.959 & 0.000 & 8771 & Optimal & 40507279.264 & 24 \\
\hline n14p6_0.9_1 & 42934170.429 & 9 & 6991.121 & 0.000 & 7914 & Optimal & 42934170.429 & 24 \\
\hline n15p6_0.6_0.8 & 42721023.156 & 7 & 58676.703 & 0.000 & 17714 & Optimal & 42721023.156 & 47 \\
\hline n15p6_0.8_0.9 & 48534512.566 & 14 & 86405.381 & 4.848 & 19267 & Feasible & 46181503.431 & 59 \\
\hline n15p6_0.9_1 & 50737550.187 & 8 & 78024.244 & 0.000 & 33688 & Optimal & 50737550.187 & 44 \\
\hline n15p7_0.6_0.8 & 40866011.253 & 9 & 11010.160 & 0.000 & 9771 & Optimal & 40866011.253 & 0 \\
\hline n15p7_0.8_0.9 & 45711471.247 & 8 & 7544.630 & 0.000 & 4825 & Optimal & 45711471.247 & 0 \\
\hline n15p7_0.9_1 & 48506492.760 & 10 & 4706.846 & 0.000 & 3577 & Optimal & 48506492.760 & 0 \\
\hline n20p6_0.6_0.8 & 84052376.665 & 2 & 86408.736 & 40.104 & 377 & Feasible & 50343311.492 & 92 \\
\hline n20p6_0.8_0.9 & 91036839.818 & 6 & 86410.608 & 32.523 & 318 & Feasible & 61428873.974 & 50 \\
\hline n20p6_0.9_1 & 94125937.923 & 6 & 86411.965 & 26.786 & 642 & Feasible & 68912769.711 & 66 \\
\hline n25p7_0.6_0.8 & - & 0 & 86400.794 & $\infty$ & 52 & AbortTimeLim & 47856545.739 & 0 \\
\hline n25p7_0.8_0.9 & 137309250.562 & 1 & 86404.304 & 56.389 & 45 & Feasible & 59881449.529 & 14 \\
\hline n25p7_0.9_1 & - & 0 & 86401.029 & $\infty$ & 57 & AbortTimeLim & 65785424.857 & 5 \\
\hline n30p8_0.6_0.8 & - & 0 & 86402.557 & $\infty$ & 27 & AbortTimeLim & 47775456.176 & 0 \\
\hline n30p8_0.8_0.9 & - & 0 & 86402.089 & $\infty$ & 23 & AbortTimeLim & 60085139.739 & 0 \\
\hline n30p8_0.9_1 & - & 0 & 86402.542 & $\infty$ & 24 & AbortTimeLim & 66130965.810 & 0 \\
\hline n35p8_0.6_0.8 & - & 0 & 86405.147 & $\infty$ & 8 & AbortTimeLim & 46776775.958 & 0 \\
\hline n35p8_0.8_0.9 & - & 0 & 86417.112 & $\infty$ & 7 & AbortTimeLim & 58880661.267 & 0 \\
\hline n35p8_0.9_1 & - & - & - & - & - & Failed & - & - \\
\hline n40p8_0.6_0.8 & - & - & - & - & - & Failed & - & - \\
\hline n40p8_0.8_0.9 & - & - & - & - & - & Failed & - & - \\
\hline n40p8_0.9_1 & - & - & - & - & - & Failed & - & - \\
\hline
\end{tabular}

the "GAP" column is calculated using the best-known solution of the hyper-heuristic and best-known solution of VNS (a negative GAP means that VNS gave a better solution than the hyper-heuristic).

The time limit $T_{\text {limit }}$ was set to $600 \mathrm{~s}(10 \mathrm{~min})$. For the hyper-heuristic, the parameter $R_{l}$ is set to 3 (number of consecutive call limit) and $d$ is set to 2 (i.e. the perturbation heuristic is called two times before reaching the stopping criterion).

The main difference between the two approaches is that in VNS, the change of neighborhood structure does not rely on any learning mechanism and each time a neighborhood is no longer capable of improving a solution, changing neighborhood $i$ to the next neighborhood $i+1$ is done without taking into account any information 


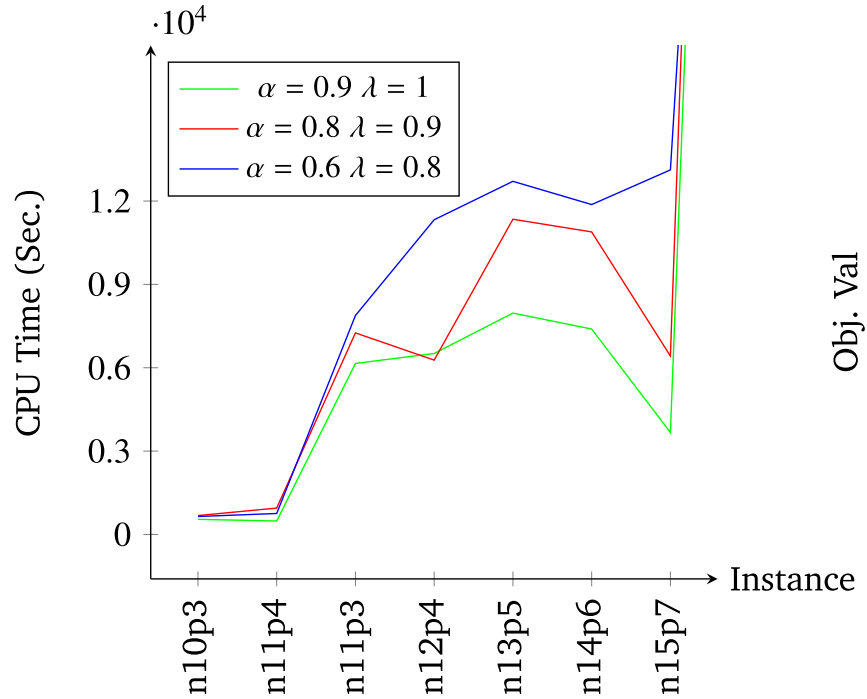

(a)

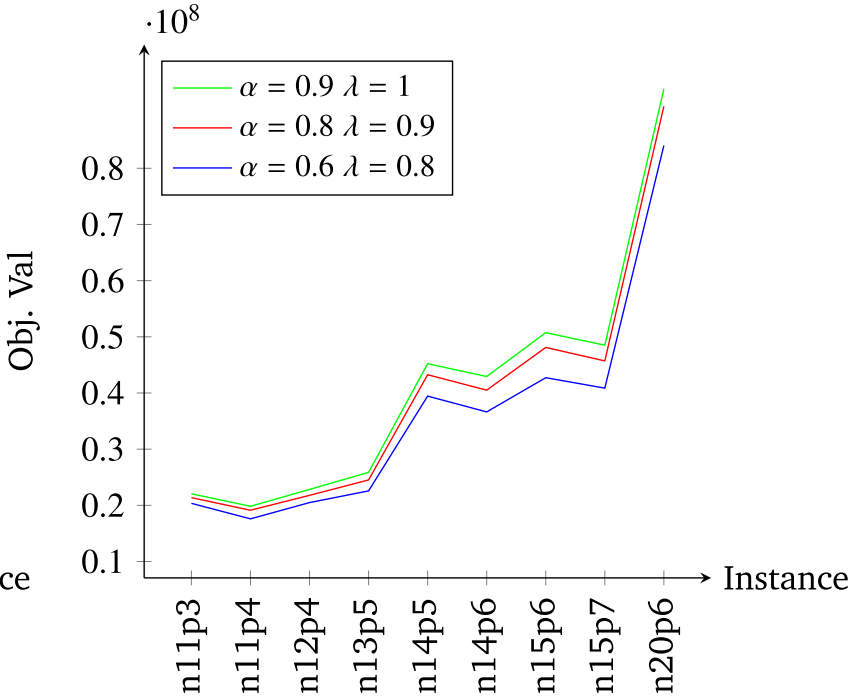

(b)

Figure 7. Numerical results of the MIP. (a) MIP time. (b) Objetive value comparison.

gathering or memory usage. On the other hand, the hyper-heuristic uses a learning mechanism to allow the overall process of algorithm to evolve with each iteration and to choose each time the best low-level heuristic to apply.

One can conclude from Table 4 that the solutions obtained by the hyper-heuristic are in general better than those of VNS, since VNS reaches the optimal solution only once (i.e. for n10p3_0.8_0.9) and the maximum gap recorded among all instances is equal to $5.877 \%$, while the hyper-heuristic fails only once in finding the optimal solution (see n13p5_0.8_0.9) and there the gap is equal to $0.003 \%$. The hyper-heuristic approach always finds a solutions better than the feasible solutions returned by CPLEX and in such a case the minimal gap recorded is equal to $-41.261 \%$.

The Table 6 elaborates on the solution structures obtained by the hyper-heuristic for medium and large size instances where "S.N." and "H.N." are the smallest and highest number of nodes per cycle, respectively. The ratio between the smallest and highest number of nodes and the average nodes per cycle $(n / p)$ are given in $"(\mathrm{~S} . \mathrm{N} . \times p) / n "$ and " $(\mathrm{H} . \mathrm{N} . \times p) / n$ ", respectively (if $(\mathrm{S} . \mathrm{N} . \times p) / n=($ H.N. $\times p) / n$, then the number of nodes associated with each hub is similar). The column "Hub Nodes" depicts the hub nodes obtained for each instance. The hub level network structure given in column "H. Net" where "C" stands for a complete graph and "NC" for incomplete graph.

Generally speaking in our obtained solutions structures (Tab. 6), there is always a hub with a high number of spokes allocated to and a hub with a low number of spokes allocated to (no solution had the same number of spokes for each hub). We observed that the hub-level network is rarely a complete graph and that the hub nodes change with $\alpha$ and $\lambda$, which shows the impact of $\alpha$ and $\lambda$ on the network structure.

Figure 8a shows that the computational time increases as instance size grows. On the other hand, the lower $\alpha$ and $\lambda$ are, the less is the computational time needed for large size instances.

The objective value for each instance of the same size should decrease with the decreasing $\alpha$ and $\lambda$ but we notice that for large instances this is not the case (see Fig. 8b). Knowing that the hyper-heuristic is deterministic and as such the algorithm follows the same path at each execution, one may conclude that if we find a value with a certain $\alpha$ and $\lambda$, we might find a better value with smaller $\alpha$ and $\lambda$ but it is not really the case. As we 
TABLE 4. Results of the hyper-heuristic and the VNS algorithms for small size instances.

\begin{tabular}{|c|c|c|c|c|c|c|c|c|}
\hline \multirow[b]{2}{*}{ Instance } & \multicolumn{4}{|c|}{ H-HLPwR } & \multicolumn{4}{|c|}{ VNS-HLPwR } \\
\hline & Obj. Val. & NSols. & E.T. (s) & $\begin{array}{l}\text { Hyper } \\
\text { cplex } \\
\operatorname{GAP}(\%)\end{array}$ & Obj. Val. & NSols. & E.T. (s) & $\begin{array}{l}\text { VNS } \\
\text { CPLEX } \\
\text { GAP (\%) }\end{array}$ \\
\hline n10p3_0.6_0.8 & 77593660.780 & 195474 & 25.483 & 0.000 & 78951975.770 & 228597 & 8.596 & 1.750 \\
\hline n10p3_0.8_0.9 & 82365794.641 & 133999 & 14.252 & 0.000 & 82365794.641 & 244174 & 9.298 & 0.000 \\
\hline n10p3_0.9_1 & 85258929.966 & 138029 & 20.347 & 0.000 & 85496741.889 & 242701 & 9.220 & 0.278 \\
\hline n11p3_0.6_0.8 & 20358494.269 & 244826 & 37.637 & 0.000 & 20449738.680 & 273972 & 12.202 & 0.448 \\
\hline n11p3_0.8_0.9 & 21375615.575 & 285467 & 43.101 & 0.000 & 21605841.250 & 283294 & 12.507 & 1.077 \\
\hline n11p3_0.9_1 & 22051911.670 & 181412 & 25.000 & 0.000 & 22057943.393 & 316949 & 14.218 & 0.027 \\
\hline n11p4_0.6_0.8 & 17597082.029 & 139594 & 20.934 & 0.000 & 17607279.345 & 291906 & 12.814 & 0.057 \\
\hline n11p4_0.8_0.9 & 19128595.920 & 121142 & 19.751 & 0.000 & 20044347.146 & 291449 & 12.869 & 4.787 \\
\hline n11p4_0.9_1 & 19835503.663 & 168888 & 24.797 & 0.000 & 20133884.670 & 309126 & 13.679 & 1.504 \\
\hline n12p44_0.6_0.8 & 20492068.237 & 219046 & 30.155 & 0.000 & 20511547.066 & 343459 & 17.670 & 0.095 \\
\hline n12p4_0.8_0.9 & 21780501.640 & 254423 & 36.231 & 0.000 & 21875191.009 & 349674 & 18.175 & 0.434 \\
\hline n12p4_0.9_1 & 22833868.689 & 151099 & 23.888 & 0.000 & 23059461.255 & 352199 & 17.891 & 0.987 \\
\hline n13p5_0.6_0.8 & 22572799.697 & 321806 & 103.566 & 0.000 & 22573390.712 & 426627 & 25.768 & 0.002 \\
\hline n13p5_0.8_0.9 & 24524102.513 & 162854 & 27.975 & 0.003 & 25063539.424 & 452671 & 26.931 & 2.203 \\
\hline n13p5_0.9_1 & 25878687.968 & 264994 & 44.231 & 0.000 & 26122787.882 & 413235 & 24.779 & 0.943 \\
\hline n14p5_0.6_0.8 & 39613147.399 & 373042 & 26.972 & -0.017 & 40151760.535 & 252445 & 17.786 & 1.341 \\
\hline n14p5_0.8_0.9 & 43252132.757 & 330950 & 45.807 & -0.212 & 45891712.452 & 241370 & 17.015 & 5.877 \\
\hline n14p5_0.9_1 & 45209210.686 & 802421 & 121.697 & 0.000 & 45724316.173 & 478423 & 33.085 & 1.139 \\
\hline n14p6_0.6_0.8 & 36633192.842 & 153804 & 28.315 & 0.000 & 36924259.216 & 347141 & 24.675 & 0.794 \\
\hline n14p6_0.8_0.9 & 40507279.264 & 196164 & 33.912 & 0.000 & 40714563.974 & 311890 & 21.565 & 0.511 \\
\hline n14p6_0.9_1 & 42934170.429 & 155273 & 25.361 & 0.000 & 43386641.062 & 322208 & 22.180 & 1.053 \\
\hline n15p6_0.6_0.8 & 42721023.156 & 353664 & 29.042 & 0.000 & 44480326.779 & 354487 & 28.907 & 4.118 \\
\hline n15p6_0.8_0.9 & 48129799.470 & 239356 & 26.547 & -0.833 & 48537186.317 & 350375 & 28.715 & 0.005 \\
\hline n15p6_0.9_1 & 50737550.187 & 221696 & 25.756 & 0.000 & 50826302.243 & 335377 & 27.480 & 0.174 \\
\hline n15p7_0.6_0.8 & 40866011.253 & 355698 & 28.966 & 0.000 & 42345232.627 & 419349 & 33.688 & 3.619 \\
\hline n15p7_0.8_0.9 & 45711471.247 & 103437 & 17.140 & 0.000 & 47162507.343 & 417507 & 34.280 & 3.174 \\
\hline n15p7_0.9_1 & 48506492.760 & 274968 & 29.948 & 0.000 & 49589687.830 & 432517 & 34.716 & 2.233 \\
\hline n20p6_0.6_0.8 & 73507081.315 & 259064 & 41.472 & -12.546 & 82417191.184 & 103146 & 15.603 & -1.945 \\
\hline n20p6_0.8_0.9 & 81841623.929 & 112504 & 18.744 & -10.100 & 83359922.804 & 124391 & 19.141 & -8.432 \\
\hline n20p6_0.9_1 & 85614920.755 & 304312 & 47.851 & -9.042 & 88920174.914 & 104588 & 16.196 & -5.530 \\
\hline n25p7_0.8_0.9 & 80654058.676 & 133604 & 38.692 & -41.261 & 83149139.101 & 97433 & 26.715 & -39.443 \\
\hline
\end{tabular}

see with the case $\alpha=0.8$ and $\lambda=0.9$, the algorithm has more difficulties to find a good solution and got stuck in a local optimum.

Figure 9a shows that the larger the instance size is, the more time consuming is the hyper-heuristic compared to VNS before it terminates, which is an indication that the hyper-heuristic does not get stuck easily in local optimum and so the mechanisms added to the hyper-heuristic to avoid the local optimum works quite well.

In terms of number of feasible solutions found, VNS is better than hyper-heuristic since in $66 \%$ of the cases it finds more solutions due to the VNS intensification heuristic, which goes deeper on every search space area (see Fig. 9b). However in terms of solution quality, which is an important aspect of the comparison, Figure 9c depicts that the hyper-heuristic finds better solutions than VNS and is more reliable and stable. In fact, the hyper-heuristic delivers good solutions on most of the instances while having found less solutions than VNS.

Table 5 gives an overview of the results of the hyper-heuristic and VNS for large solution space, where finding a promising area containing eventually the optimal solution becomes difficult. We observe that the highest gaps are recorded for large size instances with large solution space. This gaps are due to the fact that the hyperheuristic chooses the promising area to explore based on knowledge obtained from the low-level heuristic quality. In other terms, the hyper-heuristic is smarter than VNS, which is based on no learning mechanism. 
TABLE 5. Results of the hyper-heuristic and the VNS algorithms for medium and large size instances.

\begin{tabular}{|c|c|c|c|c|c|c|c|}
\hline \multirow[b]{2}{*}{ Instance } & \multicolumn{3}{|c|}{ H-HLPwR } & \multicolumn{3}{|c|}{ VNS-HLPwR } & \multirow[b]{2}{*}{$\begin{array}{l}\text { Hyper } \\
\text { VNS } \\
\text { GAP } \\
(\%) \\
\end{array}$} \\
\hline & Obj. Val. & NSols. & E.T. (s) & Obj. Val. & NSols. & E.T. (s) & \\
\hline n40p4_0.6_0.8 & 148138786.090 & 49401 & 49.286 & 149117126.105 & 67801 & 68.441 & 0.660 \\
\hline n40p4_0.8_0.9 & 161114461.028 & 58541 & 57.061 & 171562517.275 & 43309 & 44.606 & 6.484 \\
\hline n40p4_0.9_1 & 161968384.722 & 69170 & 68.254 & 167445833.970 & 75164 & 74.924 & 3.381 \\
\hline n40p5_0.6_0.8 & 113005244.011 & 115219 & 111.825 & 128879209.738 & 64899 & 66.036 & 14.047 \\
\hline n40p5_0.8_0.9 & 131221540.285 & 121454 & 115.098 & 139019009.543 & 73150 & 73.674 & 5.942 \\
\hline n40p5_0.9_1 & 154162076.939 & 52450 & 60.923 & 160013968.489 & 68049 & 62.954 & 3.795 \\
\hline n40p6_0.6_0.8 & 103984445.129 & 55506 & 54.058 & 106714188.198 & 62957 & 63.155 & 2.625 \\
\hline n40p6_0.8_0.9 & 104767544.042 & 89533 & 87.950 & 108889345.755 & 77408 & 78.165 & 3.934 \\
\hline n40p6_0.9_1 & 114734524.101 & 58083 & 57.155 & 113796258.989 & 80747 & 80.090 & -0.817 \\
\hline n50p4_0.6_0.8 & 157909765.531 & 14552 & 27.030 & 169438116.289 & 21700 & 38.844 & 7.300 \\
\hline n50p4_0.8_0.9 & 159532981.095 & 13617 & 25.428 & 171028682.989 & 19643 & 34.615 & 7.205 \\
\hline n50p4_0.9_1 & 158102762.268 & 20520 & 37.662 & 174131059.797 & 29592 & 52.548 & 10.137 \\
\hline n50p5_0.6_0.8 & 134342531.901 & 22852 & 42.470 & 137296304.548 & 26579 & 48.386 & 2.198 \\
\hline n50p5_0.8_0.9 & 141056845.686 & 32446 & 58.329 & 154072647.019 & 24924 & 44.502 & 9.227 \\
\hline n50p5_0.9_1 & 143075031.912 & 90191 & 156.127 & 143168823.947 & 106488 & 186.436 & 0.065 \\
\hline n50p6_0.6_0.8 & 111256527.176 & 74433 & 119.888 & 117844078.512 & 77151 & 122.628 & 5.921 \\
\hline n50p6_0.8_0.9 & 121089305.695 & 68729 & 110.447 & 130724405.366 & 36941 & 64.917 & 7.957 \\
\hline n50p6_0.9_1 & 125815623.235 & 83985 & 136.744 & 128589484.436 & 45055 & 81.393 & 2.204 \\
\hline n60p4_0.6_0.8 & 169002097.262 & 42159 & 108.108 & 173947891.806 & 35096 & 98.594 & 2.926 \\
\hline n60p4_0.8_0.9 & 175188580.124 & 24738 & 65.251 & 187725676.808 & 56915 & 158.611 & 7.156 \\
\hline n60p4_0.9_1 & 178425530.893 & 43249 & 112.677 & 187685325.418 & 35681 & 98.582 & 5.189 \\
\hline n60p5_0.6_0.8 & 154967528.427 & 23333 & 73.805 & 160569265.881 & 55401 & 160.104 & 3.614 \\
\hline n60p5_0.8_0.9 & 162381946.539 & 73275 & 210.158 & 157765154.746 & 51213 & 151.268 & -2.843 \\
\hline n60p5_0.9_1 & 166500564.721 & 43549 & 131.203 & 167795253.727 & 39734 & 116.069 & 0.777 \\
\hline n60p6_0.6_0.8 & 122545418.790 & 77638 & 226.746 & 145232971.584 & 88203 & 223.523 & 18.513 \\
\hline n60p6_0.8_0.9 & 146113509.668 & 41377 & 128.693 & 142914535.634 & 46197 & 136.241 & -2.189 \\
\hline n60p6_0.9_1 & 147703870.562 & 29562 & 92.513 & 161694330.738 & 41292 & 119.069 & 9.471 \\
\hline n70p4_0.6_0.8 & 192428066.145 & 28486 & 138.032 & 179045038.186 & 40338 & 179.023 & -6.954 \\
\hline n70p4_0.8_0.9 & 206603867.306 & 34696 & 172.072 & 228474586.016 & 36444 & 160.256 & 10.585 \\
\hline n70p4_0.9_1 & 224829414.796 & 28259 & 130.518 & 274812800.508 & 25042 & 108.964 & 22.231 \\
\hline n70p5_0.6_0.8 & 149207903.263 & 112073 & 438.122 & 161857851.826 & 151284 & 578.071 & 8.478 \\
\hline n70p5_0.8_0.9 & 161817036.066 & 79393 & 315.656 & 183353913.235 & 41039 & 186.520 & 13.309 \\
\hline n70p5_0.9_1 & 187921152.051 & 100723 & 414.303 & 193965901.238 & 69184 & 261.476 & 3.216 \\
\hline n70p6_0.6_0.8 & 174772974.482 & 35851 & 163.751 & 158446540.981 & 49174 & 202.502 & -9.341 \\
\hline n70p6_0.8_0.9 & 171566552.198 & 39451 & 197.199 & 188350564.183 & 46674 & 214.103 & 9.782 \\
\hline n70p6_0.9_1 & 158806582.863 & 46254 & 221.485 & 167322855.988 & 73401 & 278.733 & 5.362 \\
\hline n80p4_0.6_0.8 & 202846715.417 & 41299 & 283.303 & 191264990.591 & 59527 & 372.048 & -5.709 \\
\hline n80p4_0.8_0.9 & 218864328.032 & 41299 & 292 & 240718670.313 & 55967 & 354.896 & 9.985 \\
\hline n80p4_0.9_1 & 220342768.129 & 71331 & 475.963 & 265826532.426 & 46223 & 278.956 & 20.642 \\
\hline n80p5_0.6_0.8 & 188885325.674 & 45660 & 314.367 & 170681155.371 & 46211 & 285.240 & -9.637 \\
\hline n80p5_0.8_0.9 & 180485726.339 & 65814 & 440.213 & 199346133.417 & 41350 & 275.257 & 10.449 \\
\hline n80p5_0.9_1 & 170580133.611 & 64659 & 429. & 180860955.637 & 98915 & 541.556 & 6.026 \\
\hline n80p6_0.6_0.8 & 183101479.379 & 26980 & 170.270 & 187349115.698 & 58557 & 350.209 & 2.319 \\
\hline
\end{tabular}


TABLE 5. continued.

\begin{tabular}{llllllll}
\hline \hline & \multicolumn{3}{c}{ H-HLPwR } & \multicolumn{3}{c}{ VNS-HLPwR } & \\
\cline { 2 - 7 } Instance & Obj. Val. & NSols. & E.T. (s) & Obj. Val. & NSols. & E.T. (s) & Hyper \\
& & & & & & & VNS \\
& & & & & & & GAP \\
& & & & & & & \\
\hline n80p6_0.8_0.9 & 183912599.384 & 75699 & 490.697 & 177529525.433 & 67853 & 432.838 & -3.470 \\
n80p6_0.9_1 & 156996820.272 & 75653 & 496.245 & 181233695.779 & 70842 & 437.536 & 15.437 \\
n90p4_0.6_0.8 & 202791891.331 & 41883 & 360.565 & 217394590.144 & 40908 & 331.785 & 7.200 \\
n90p4_0.8_0.9 & 224482869.606 & 51825 & $T_{\text {limit }}$ & 223106286.458 & 57486 & 473.120 & -0.613 \\
n90p4_0.9_1 & 229610546.982 & 55191 & 533.389 & 226589361.793 & 51524 & 422.694 & -1.315 \\
n90p5_0.6_0.8 & 166226023.566 & 64500 & $T_{\text {limit }}$ & 207363553.578 & 79885 & $T_{\text {limit }}$ & 24.747 \\
n90p5_0.8_0.9 & 184094110.772 & 28865 & 275.663 & 185995172.129 & 73340 & $T_{\text {limit }}$ & 1.032 \\
n90p5_0.9_1 & 167416557.335 & 66038 & 591.735 & 207381153.700 & 54811 & 454.516 & 23.871 \\
n90p6_0.6_0.8 & 165366131.295 & 64900 & $T_{\text {limit }}$ & 193071250.058 & 78046 & $T_{\text {limit }}$ & 16.753 \\
n90p6_0.8_0.9 & 200491533.112 & 64651 & $T_{\text {limit }}$ & 194814834.654 & 63863 & 526.826 & -2.831 \\
n90p6_0.9_1 & 195582760.605 & 66573 & $T_{\text {limit }}$ & 182757300.005 & 72799 & $T_{\text {limit }}$ & -6.557 \\
n100p4_0.6_0.8 & 214772996.844 & 52494 & $T_{\text {limit }}$ & 212171192.914 & 53987 & 575.176 & -1.211 \\
n100p4_0.8_0.9 & 267448010.993 & 59359 & $T_{\text {limit }}$ & 256225646.356 & 58265 & $T_{\text {limit }}$ & -4.196 \\
n100p4_0.9_1 & 219585842.209 & 48640 & $T_{\text {limit }}$ & 255975181.789 & 55463 & 588.572 & 16.571 \\
n100p5_0.6_0.8 & 182589034.595 & 51399 & $T_{\text {limit }}$ & 207742381.588 & 46475 & 518.437 & 13.775 \\
n100p5_0.8_0.9 & 207744504.144 & 54316 & $T_{\text {limit }}$ & 220157176.003 & 56684 & 599.679 & 5.974 \\
n100p5_0.9_1 & 215090122.749 & 63343 & $T_{\text {limit }}$ & 216977957.144 & 52448 & 545.058 & 0.877 \\
n100p6_0.6_0.8 & 167022456.992 & 52533 & $T_{\text {limit }}$ & 181540345.551 & 53437 & $T_{\text {limit }}$ & 8.692 \\
n100p6_0.8_0.9 & 182849599.132 & 46595 & $T_{\text {limit }}$ & 185083903.866 & 55913 & $T_{\text {limit }}$ & 1.221 \\
n100p6_0.9_1 & 168247877.285 & 51697 & $T_{\text {limit }}$ & 194676676.274 & 55197 & $T_{\text {limit }}$ & 15.708 \\
\hline
\end{tabular}

TABLE 6. Hyper-heuristic structures information for medium and large size instances.

\begin{tabular}{lllllll}
\hline \hline Instance & S.N. & $(\mathrm{S} . \mathrm{N} . \times p) / n$ & H.N. & $($ H.N. $\times p) / n$ & Hub Nodes & H. Net. \\
\hline n40p4_0.6_0.8 & 6 & 0.60 & 11 & 1.10 & $9 ; 17 ; 2 ; 25$ & $\mathrm{C}$ \\
n40p4_0.8_0.9 & 3 & 0.30 & 13 & 1.30 & $18 ; 3 ; 11 ; 25$ & $\mathrm{NC}$ \\
n40p4_0.9_1 & 3 & 0.30 & 17 & 1.70 & $7 ; 27 ; 26 ; 25$ & $\mathrm{NC}$ \\
n40p5_0.6_0.8 & 6 & 0.75 & 10 & 1.25 & $4 ; 19 ; 30 ; 28 ; 25$ & $\mathrm{NC}$ \\
n40p5_0.8_0.9 & 5 & 0.62 & 13 & 1.62 & $1 ; 26 ; 17 ; 27 ; 34$ & $\mathrm{NC}$ \\
n40p5_0.9_1 & 4 & 0.50 & 20 & 2.50 & $1 ; 8 ; 17 ; 6 ; 9$ & $\mathrm{NC}$ \\
n40p6_0.6_0.8 & 4 & 0.60 & 10 & 1.50 & $4 ; 19 ; 30 ; 36 ; 11 ; 2$ & $\mathrm{NC}$ \\
n40p6_0.8_0.9 & 6 & 0.90 & 8 & 1.20 & $18 ; 12 ; 36 ; 26 ; 11 ; 19$ & $\mathrm{NC}$ \\
n40p6_0.9_1 & 5 & 0.75 & 10 & 1.50 & $10 ; 28 ; 35 ; 17 ; 11 ; 19$ & $\mathrm{NC}$ \\
n50p4_0.6_0.8 & 7 & 0.56 & 20 & 1.60 & $6 ; 12 ; 13 ; 35$ & $\mathrm{NC}$ \\
n50p4_0.8_0.9 & 8 & 0.64 & 18 & 1.44 & $23 ; 15 ; 13 ; 22$ & $\mathrm{NC}$ \\
n50p4_0.9_1 & 8 & 0.64 & 16 & 1.28 & $14 ; 12 ; 31 ; 22$ & $\mathrm{C}$ \\
n50p5_0.6_0.8 & 6 & 0.60 & 17 & 1.70 & $5 ; 15 ; 13 ; 31 ; 6$ & $\mathrm{NC}$ \\
n50p5_0.8_0.9 & 2 & 0.20 & 16 & 1.60 & $5 ; 23 ; 13 ; 12 ; 6$ & $\mathrm{NC}$ \\
n50p5_0.9_1 & 2 & 0.20 & 20 & 2.00 & $23 ; 31 ; 13 ; 12 ; 42$ & $\mathrm{NC}$ \\
n50p6_0.6_0.8 & 3 & 0.36 & 14 & 1.68 & $32 ; 34 ; 39 ; 33 ; 15 ; 7$ & $\mathrm{NC}$ \\
n50p6_0.8_0.9 & 4 & 0.48 & 10 & 1.20 & $12 ; 45 ; 6 ; 37 ; 36 ; 13$ & $\mathrm{NC}$ \\
n50p6_0.9_1 & 5 & 0.60 & 13 & 1.56 & $3 ; 32 ; 23 ; 31 ; 6 ; 22$ & $\mathrm{NC}$ \\
n60p4_0.6_0.8 & 2 & 0.13 & 24 & 1.60 & $26 ; 42 ; 47 ; 41$ & $\mathrm{NC}$ \\
n60p4_0.8_0.9 & 7 & 0.46 & 29 & 1.93 & $26 ; 42 ; 44 ; 8$ & $\mathrm{NC}$ \\
\hline
\end{tabular}


TABLE 6. continued.

\begin{tabular}{|c|c|c|c|c|c|c|}
\hline Instance & S.N. & $(\mathrm{S} . \mathrm{N} . \times p) / n$ & H.N. & $($ H.N. $\times p) / n$ & Hub Nodes & H. Net. \\
\hline n60p4_0.9_1 & 8 & 0.53 & 22 & 1.46 & $14 ; 40 ; 1 ; 27$ & $\mathrm{NC}$ \\
\hline n60p5_0.6_0.8 & 7 & 0.58 & 22 & 1.83 & $13 ; 41 ; 7 ; 15 ; 24$ & $\mathrm{NC}$ \\
\hline n60p5_0.8_0.9 & 3 & 0.25 & 27 & 2.25 & $13 ; 1 ; 0 ; 14 ; 2$ & $\mathrm{NC}$ \\
\hline n60p5_0.9_1 & 5 & 0.41 & 24 & 2.00 & $12 ; 1 ; 26 ; 3 ; 40$ & $\mathrm{NC}$ \\
\hline n60p6_0.6_0.8 & 8 & 0.80 & 16 & 1.60 & $29 ; 54 ; 6 ; 1 ; 41 ; 18$ & $\mathrm{NC}$ \\
\hline n60p6_0.8_0.9 & 2 & 0.20 & 18 & 1.80 & $14 ; 0 ; 6 ; 1 ; 13 ; 15$ & $\mathrm{NC}$ \\
\hline n60p6_0.9_1 & 2 & 0.20 & 17 & 1.70 & $2 ; 26 ; 14 ; 1 ; 18 ; 15$ & $\mathrm{NC}$ \\
\hline n70p4_0.6_0.8 & 5 & 0.28 & 30 & 1.71 & $38 ; 48 ; 46 ; 21$ & $\mathrm{NC}$ \\
\hline n70p4_0.8_0.9 & 8 & 0.45 & 35 & 2.00 & $55 ; 48 ; 3 ; 31$ & $\mathrm{NC}$ \\
\hline n70p4_0.9_1 & 9 & 0.51 & 38 & 2.17 & $10 ; 48 ; 53 ; 34$ & $\mathrm{C}$ \\
\hline n70p5_0.6_0.8 & 9 & 0.64 & 25 & 1.78 & $8 ; 48 ; 3 ; 21 ; 46$ & $\mathrm{NC}$ \\
\hline n70p5_0.8_0.9 & 6 & 0.42 & 26 & 1.85 & $8 ; 48 ; 18 ; 4 ; 63$ & $\mathrm{NC}$ \\
\hline n70p5_0.9_1 & 6 & 0.42 & 26 & 1.85 & $6 ; 5 ; 20 ; 45 ; 46$ & $\mathrm{NC}$ \\
\hline n70p6_0.6_0.8 & 6 & 0.51 & 21 & 1.80 & $38 ; 48 ; 18 ; 3 ; 46 ; 34$ & $\mathrm{NC}$ \\
\hline n70p6_0.8_0.9 & 5 & 0.42 & 20 & 1.71 & $10 ; 44 ; 2 ; 43 ; 31 ; 19$ & $\mathrm{NC}$ \\
\hline n70p6_0.9_1 & 3 & 0.25 & 25 & 2.14 & $21 ; 15 ; 2 ; 3 ; 6 ; 14$ & $\mathrm{NC}$ \\
\hline n80p4_0.6_0.8 & 2 & 0.10 & 37 & 1.85 & $54 ; 78 ; 23 ; 22$ & $\mathrm{NC}$ \\
\hline n80p4_0.8_0.9 & 6 & 0.30 & 39 & 1.95 & $54 ; 53 ; 8 ; 21$ & C \\
\hline n80p4_0.9_1 & 8 & 0.40 & 39 & 1.95 & $54 ; 71 ; 8 ; 7$ & $\mathrm{C}$ \\
\hline n80p5_0.6_0.8 & 2 & 0.12 & 31 & 1.93 & $54 ; 3 ; 6 ; 21 ; 71$ & $\mathrm{NC}$ \\
\hline n80p5_0.8_0.9 & 13 & 0.81 & 20 & 1.25 & $49 ; 53 ; 36 ; 35 ; 37$ & $\mathrm{NC}$ \\
\hline n80p5_0.9_1 & 13 & 0.81 & 22 & 1.37 & $54 ; 57 ; 52 ; 33 ; 20$ & $\mathrm{NC}$ \\
\hline n80p6_0.6_0.8 & 4 & 0.30 & 31 & 2.32 & $49 ; 6 ; 63 ; 1 ; 4 ; 33$ & $\mathrm{NC}$ \\
\hline n80p6_0.8_0.9 & 8 & 0.60 & 22 & 1.65 & $49 ; 11 ; 35 ; 2 ; 22 ; 18$ & $\mathrm{NC}$ \\
\hline n80p6_0.9_1 & 7 & 0.52 & 24 & 1.80 & $56 ; 54 ; 35 ; 2 ; 22 ; 52$ & $\mathrm{NC}$ \\
\hline n90p4_0.6_0.8 & 15 & 0.66 & 37 & 1.64 & $23 ; 10 ; 55 ; 25$ & $\mathrm{NC}$ \\
\hline n90p4_0.8_0.9 & 11 & 0.48 & 40 & 1.77 & $4 ; 10 ; 18 ; 19$ & $\mathrm{NC}$ \\
\hline n90p4_0.9_1 & 11 & 0.48 & 32 & 1.42 & $50 ; 10 ; 55 ; 24$ & $\mathrm{NC}$ \\
\hline n90p5_0.6_0.8 & 13 & 0.72 & 28 & 1.55 & $37 ; 21 ; 7 ; 8 ; 13$ & $\mathrm{NC}$ \\
\hline n90p5_0.8_0.9 & 12 & 0.66 & 24 & 1.33 & $55 ; 2 ; 8 ; 25 ; 31$ & $\mathrm{NC}$ \\
\hline n90p5_0.9_1 & 11 & 0.61 & 28 & 1.55 & $55 ; 42 ; 8 ; 39 ; 30$ & $\mathrm{NC}$ \\
\hline n90p6_0.6_0.8 & 6 & 0.40 & 30 & 2.00 & $55 ; 2 ; 18 ; 61 ; 26 ; 17$ & $\mathrm{NC}$ \\
\hline n90p6_0.8_0.9 & 3 & 0.20 & 33 & 2.20 & $55 ; 10 ; 16 ; 23 ; 44 ; 5$ & $\mathrm{NC}$ \\
\hline n90p6_0.9_1 & 5 & 0.33 & 33 & 2.20 & $55 ; 10 ; 8 ; 23 ; 38 ; 4$ & $\mathrm{NC}$ \\
\hline n100p4_0.6_0.8 & 11 & 0.44 & 41 & 1.64 & $73 ; 31 ; 6 ; 69$ & $\mathrm{NC}$ \\
\hline n100p4_0.8_0.9 & 6 & 0.24 & 54 & 2.16 & $66 ; 31 ; 32 ; 69$ & $\mathrm{NC}$ \\
\hline n100p4_0.9_1 & 15 & 0.6 & 41 & 1.64 & $66 ; 3 ; 29 ; 69$ & $\mathrm{NC}$ \\
\hline n100p5_0.6_0.8 & 7 & 0.35 & 36 & 1.80 & $69 ; 27 ; 77 ; 50 ; 9$ & $\mathrm{NC}$ \\
\hline n100p5_0.8_0.9 & 6 & 0.30 & 38 & 1.90 & $69 ; 27 ; 77 ; 50 ; 7$ & $\mathrm{NC}$ \\
\hline n100p5_0.9_1 & 6 & 0.30 & 37 & 1.85 & $69 ; 31 ; 13 ; 47 ; 9$ & $\mathrm{NC}$ \\
\hline n100pp_0.6_0.8 & 4 & 0.24 & 29 & 1.74 & $6 ; 48 ; 9 ; 47 ; 44 ; 7$ & $\mathrm{NC}$ \\
\hline n100p6_0.8_0.9 & 4 & 0.24 & 33 & 1.98 & $10 ; 48 ; 12 ; 46 ; 44 ; 7$ & $\mathrm{NC}$ \\
\hline n100p6_0.9_1 & 4 & 0.24 & 26 & 1.56 & $6 ; 21 ; 9 ; 47 ; 27 ; 68$ & $\mathrm{NC}$ \\
\hline
\end{tabular}




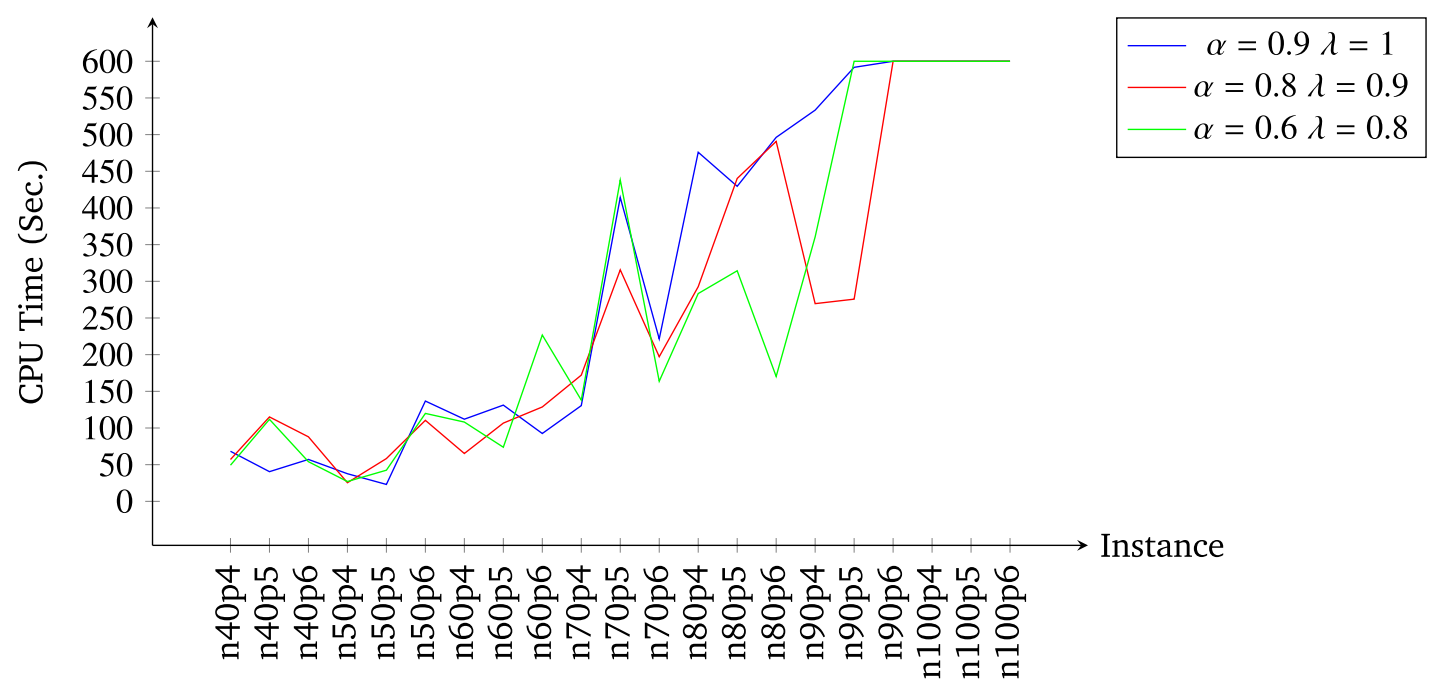

(a)

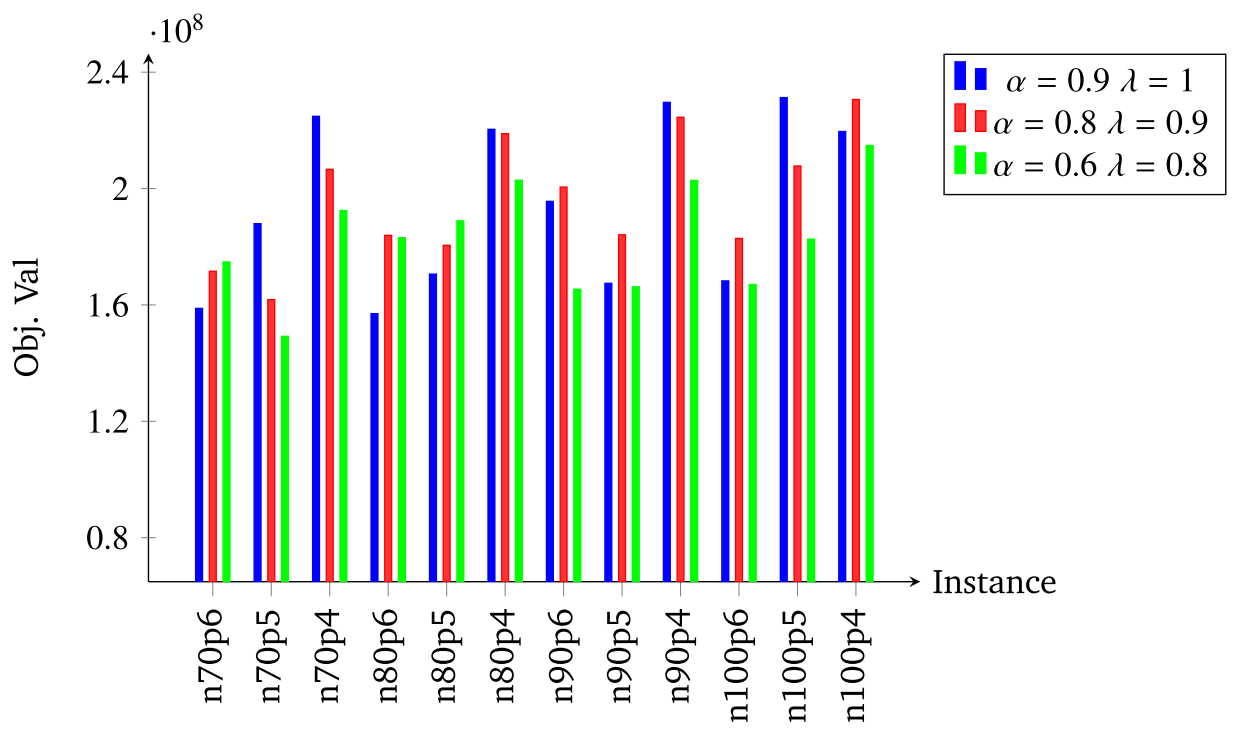

(b)

Figure 8. Numerical results of the H-HLPwR. (a) Execution time. (b) Objective value.

Our hyper-heuristic algorithm has demonstrated its effectiveness both in solution quality and computational time even for larger size instances when compared to comparable methods.

\section{Summary AND CONCLUSION}

In this study, we have introduced a new variant of the hub location problem that we referred to as $p$-Hub Location Problem with Runaway. The runaway nodes introduced in this work are of special interest in service networks based on round-trips where a set of nodes being served by one single hub node whose failure can disrupt 


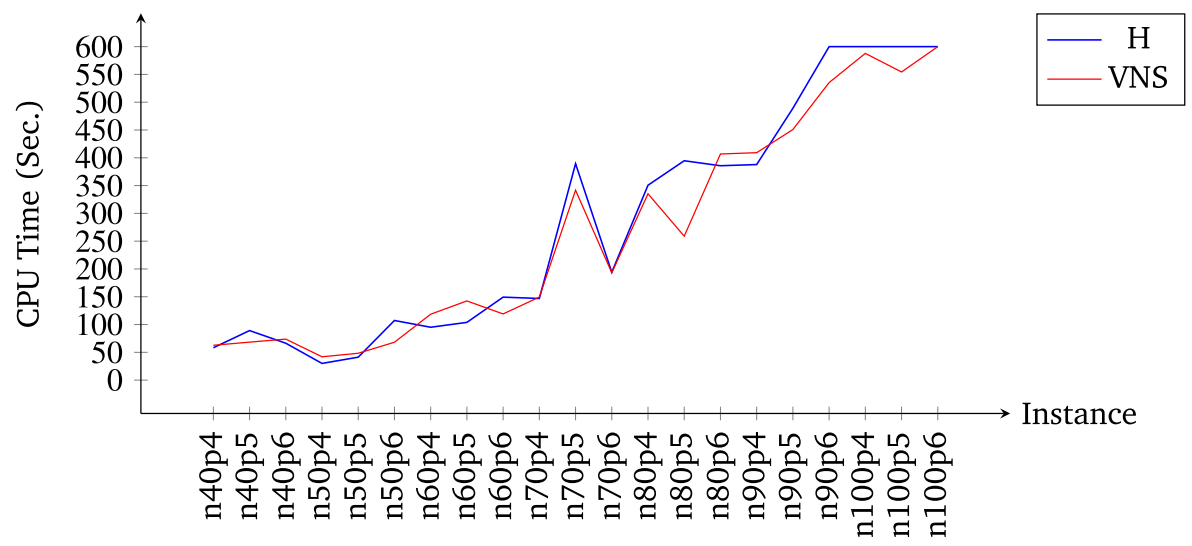

(a)

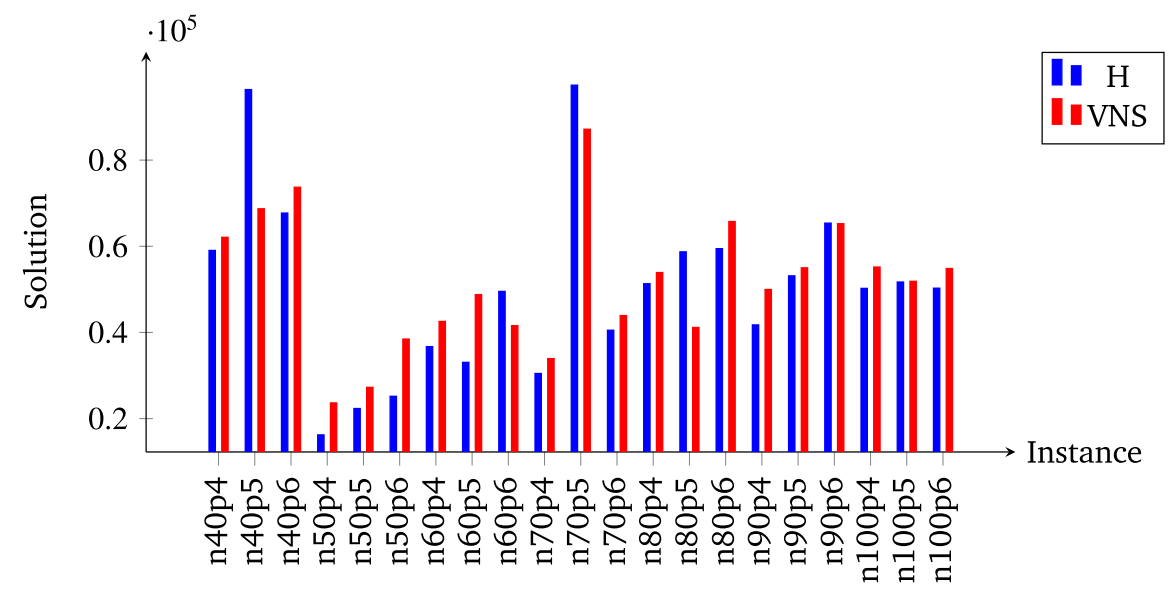

(b)

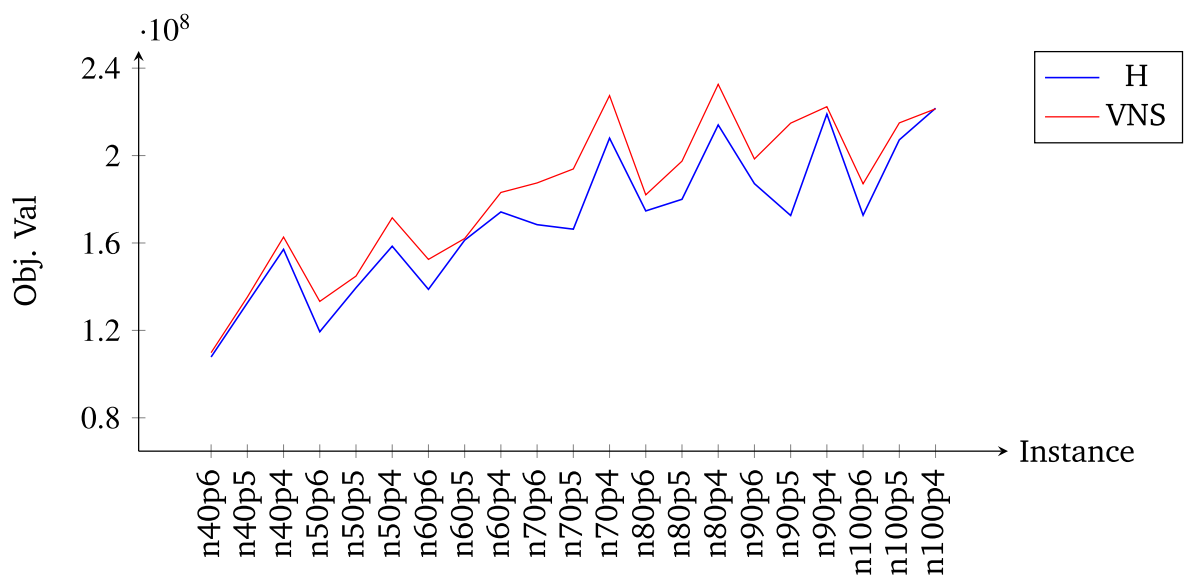

(c)

FiguRE 9. Numerical results comparison between the hyper-heuristic and VNS. (a) Time comparison. (b) Number of solutions found. (c) Objective value comparison. 
an important part of the supply network. Based on what is being practiced in real life, we have proposed a mathematical model of this problem.

For the proposed mathematical model with exponential number of constraints, we provided separation routines to separate from such constraints in a branch-and-cut manner in CPLEX. Yet, due to the complexity of the problem, CPLEX can only tackle small size instances. We therefore proposed a hyper-heuristic approach for our problem. This approach consists of a set of low-level heuristics and a selection heuristic. The objective of the selection heuristic is to choose the best low-level heuristic to apply at different phases in order to find the best solutions. Our selection heuristic is guided by a reinforcement learning method based on a scoring system. We also improved the efficiency of our hyper-heuristic by adding a powerful perturbation heuristic and a tabu list. In order to be as close as possible to a fair comparison of the hyper-heuristic results, we proposed a VNS meta-heuristic implemented using the same hyper-heuristic low-level heuristics and components.

Our computational experiments confirm that whenever the optimal solution is known, the hyper-heuristic is almost always able to find it in much less computational time. On the other hand, the VNS approach, which inherits the same local search methods, seldom finds an optimal solution. The two approaches find feasible solutions in a reasonable amount of time but the absolute superiority remains with hyper-heuristic both for quality and computational time.

Further work directions include polyhedral analysis, identifying some tightening valid inequalities to improve the polyhedral description and primal decompositions. Furthermore, as the hyper-heuristic shown to be promising, more enhanced learning mechanisms, designing a richer portfolio of low-level heuristics and more sophisticated selection methods deserve more attention.

Acknowledgements. The authors would like to thank the Directorate General of Scientific Research and Technological Development (DGRSDT), Ministry of Higher Education and Scientific Research for their support in this work. In addition, work of last author has been supported by the PGMO, the Gaspard Monge Program for Optimisation and Operational Research in the framework of BENMIP project.

\section{REFERENCES}

[1] S.A. Alumur, H. Yaman and B.Y. Kara, Hierarchical multimodal hub location problem with time-definite deliveries. Transp. Res. Part E: Logistics Transp. Rev. 48 (2012) 1107-1120.

[2] N. Azizi, Managing facility disruption in hub-and-spoke networks: formulations and efficient solution methods. Ann. Oper. Res. 272 (2019) 159-185.

[3] O. Berman, Z. Drezner and G.O. Wesolowsky, The transfer point location problem. Eur. J. Oper. Res. 179 (2007) $978-989$.

[4] E.K. Burke, M. Gendreau, M. Hyde, G. Kendall, G. Ochoa, E. Özcan and R. Qu, Hyper-heuristics: a survey of the state of the art. J. Oper. Res. Soc. 64 (2013) 1695-1724.

[5] J.F. Campbell, Location and allocation for distribution systems with transshipments and transportion economies of scale. Ann. Oper. Res. 40 (1992) 77-99.

[6] J.F. Campbell, Integer programming formulations of discrete hub location problems. Eur. J. Oper. Res. 72 (1994) $387-405$.

[7] S.R. Cardoso, A.P. Barbosa-Póvoa, S. Relvas and A.Q. Novais, Resilience metrics in the assessment of complex supply-chains performance operating under demand uncertainty. Omega 56 (2015) 53-73.

[8] G. Carello, F.D. Croce, M. Ghirardi and R. Tadei, Solving the hub location problem in telecommunication network design: a local search approach. Networks 44 (2004) 94-105.

[9] P. Carroll, B. Fortz, M. Labbé and S. McGarraghy, Improved formulations for the ring spur assignment problem, in Network Optimization. INOC 2011, edited by J. Pahl, T. Reiners and S. Voß. Vol. 6701 of Lecture Notes in Computer Science. Springer, Berlin-Heidelberg (2011) 24-36.

[10] S. Çetiner, C. Sepil and H. Süral, Hubbing and routing in postal delivery systems. Ann. Oper. Res. 181 (2010) $109-124$.

[11] S. Chaharsooghi, F. Momayezi and N. Ghaffarinasab, An adaptive large neighborhood search heuristic for solving the reliable multiple allocation hub location problem under hub disruptions. Int. J. Ind. Eng. Comput. 8 (2016) 191-202.

[12] I. Contreras, M. Tanash and N. Vidyarthi, Exact and heuristic approaches for the cycle hub location problem. Ann. Oper. Res. 258 (2017) 655-677.

[13] P.I. Cowling, G. Kendall and E. Soubeiga, A hyperheuristic approach to scheduling a sales summit. In: Practice and Theory of Automated Timetabling III, PATAT '00. Springer (2001) 176-190.

[14] W. Dai, J. Zhang, X. Sun and S. Wandelt, Hubbi: iterative network design for incomplete hub location problems. Comput. Oper. Res. 104 (2019) 394-414.

[15] K. Danach, Hyperheuristics in Logistics. Ph.D. thesis, Ecole Centrale de Lille (2016). 
[16] K. Danach, S. Gelareh and R. Neamatian Monemi, The capacitated single-allocation p-hub location routing problem: a lagrangian relaxation and a hyper-heuristic approach. EURO J. Transp. Logistics. 8 (2019) 597-631.

[17] J. Denzinger and M. Fuchs, High performance ATP systems by combining several AI methods. In: Vol. 1 of IJCAI'97. Proceedings of the 15th International Joint Conference on Artificial Intelligence. Morgan Kaufmann Publishers Inc. (1997) 102-107.

[18] J. Ebery, M. Krishnamoorthy, A. Ernst, and N. Boland, The capacitated multiple allocation hub location problem: formulations and algorithms. Eur. J. Oper. Res. 120 (2000) 614-631.

[19] A.T. Ernst and M. Krishnamoorthy, Efficient algorithms for the uncapacitated single allocation p-hub median problem. Location Sci. 4 (1996) 139-154.

[20] S. Gelareh and S. Nickel, Hub location problems in transportation networks. Transp. Res. Part E: Logistics Transp. Rev. 47 (2011) 1092-1111.

[21] S. Gelareh, N. Maculan, P. Mahey and R.N. Monemi, Hub-and-spoke network design and fleet deployment for string planning of liner shipping. Appl. Math. Model. 37 (2013) 3307-3321.

[22] S. Gelareh, R. Neamatian Monemic and F. Semet, Capacitated bounded cardinality hub routing problem: model and solution algorithm. Technical report Preprint arXiv:1705.07985 (2017).

[23] Z. He, Farthest-point heuristic based initialization methods for $k$-modes clustering. CoRR, abs/cs/0610043 (2006).

[24] D. Huang, Z. Liu, X. Fu and P. Blythe, Multimodal transit network design in a hub-and-spoke network framework. Transp. A: Transp. Sci. 14 (2018) 706-35.

[25] B. Jarboui, H. Derbel, S. Hanafi and N. Mladenovic, Variable neighborhood search for location routing. Comput. Oper. Res. 40 (2013) 47-57.

[26] H. Kim and M. O'Kelly, Reliable p-hub location problems in telecommunication networks. Geogr. Anal. 41 (2009) $283-306$.

[27] M.J. Kuby and R.G. Gray, The hub network design problem with stopovers and feeders: the case of federal express. Transp. Res. Part A: Policy Practice 27 (1993) 1-12.

[28] J.B. MacQueen, Some methods for classification and analysis of multivariate observations. In: Vol. 1 of Proceedings of the fifth Berkeley Symposium on Mathematical Statistics and Probability. University of California Press (1967) 281-297.

[29] E. Martins de Sá, I. Contreras and J.-F. Cordeau, Exact and heuristic algorithms for the design of hub networks with multiple lines. Eur. J. Oper. Res. 246 (2015) 186-198.

[30] E. Martins de Sá, I. Contreras, J.-F. Cordeau, R. Saraiva de Camargo and G. de Miranda, The hub line location problem. Transp. Sci. 49 (2015) 500-518.

[31] N. Mladenović and P. Hansen, Variable neighborhood search. Comput. Oper. Res. 24 (1997) 1097-1100.

[32] M. Mohammadi, R. Tavakkoli-Moghaddam, A. Siadat and Y. Rahimi, A game-based meta-heuristic for a fuzzy bi-objective reliable hub location problem. Eng. App. Artif. Intel. 50 (2016) 1-19.

[33] R.N. Monemi and S. Gelareh, The ring spur assignment problem: new formulation, valid inequalities and a branch-and-cut approach. Comput. Oper. Res. 88 (2017) 91-102.

[34] S. Mourelo Ferrandez, T. Harbison, T. Weber, R. Sturges and R. Rich, Optimization of a truck-drone in tandem delivery network using $k$-means and genetic algorithm. J. Ind. Eng. Manage. 9 (2016) 374.

[35] M. O'Kelly, Hub facility location with fixed costs. Papers Regional Sci. 71 (1992) 293-306.

[36] M. O'Kelly, A clustering approach to the planar hub location problem. Ann. Oper. Res. 40 (1993) 339-353.

[37] M.P. Pérez, F.A. Rodíguez and J.M. Moreno-Vega, A hybrid VNS-path relinking for the p-hub median problem. IMA J. Manage. Math. 18 (2007) 157-171.

[38] J. Pérez-Ortega, N.A.-O. Nelva, A. Vega-Villalobos, R. Pazos-Rangel, C. Zavala-Diaz and A. Martinez-Rebollar, The K-means algorithm evolution, edited by K. Sud, P. Erdogmus and S. Kadry. In: Introduction to Data Science and Machine Learning. IntechOpen, Rijeka (2020).

[39] R. Rahmaniani, G. Rahmaniani and A. Jabbarzadeh, Variable neighborhood search based evolutionary algorithm and several approximations for balanced location-allocation design problem. Int. J. Adv. Manuf. Technol. 72 (2014) $145-159$.

[40] I. Rodriguez-Martin, J.J. Salazar González and H. Yaman, A branch-and-cut algorithm for the hub location and routing problem. Comput. Oper. Res. 50 (2014) 161-174.

[41] I. Rodriguez-Martin, J.J. Salazar González and H. Yaman, The ring $k$-rings network design problem: model and branch-and-cut algorithm. Networks 68 (2016) 130-140.

[42] B. Rostami, N. Kämmerling, C. Buchheim and U. Clausen, Reliable single allocation hub location problem under hub breakdowns. Comput. Oper. Res. 96 (2018) 15-29.

[43] E. Serper and S. Alumur Alev, The design of capacitated intermodal hub networks with different vehicle types. Transp. Res. Part B: Methodol. 86 (2016) 51-65.

[44] D. Skorin-Kapov, J. Skorin-Kapov and M. O'Kelly, Tight linear programming relaxations of uncapacitated p-hub median problems. Eur. J. Oper. Res. 94 (1996) 582-593.

[45] R. Todosijević, D. Urosevic, N. Mladenovic and S. Hanafi, A general variable neighborhood search for solving the uncapacitated r-allocation p-hub median problem. Optim. Lett. 11(2017) 1109-1121.

[46] UNCTAD, Review of maritime transport. In: United Nations Conference on Trade and Development, New York and Geneva (2018).

[47] M.Yahyaei, M. Bashiri and Y. Garmeyi, Multicriteria logistic hub location by network segmentation under criteria weights uncertainty. Int. J. Eng. Trans. B: App. 27 (2014) 1205-1214. 
[48] M. Yahyaei, M. Bashiri and M. Randall, A model for a reliable single allocation hub network design under massive disruption. Appl. Soft Comput. 82 (2019) 105561.

[49] H. Yaman, B.Y. Kara and B. Tansel, The latest arrival hub location problem for cargo delivery systems with stopovers. Transp. Res. Part B: Methodol. 41 (2007) 906-919.

[50] K. Yang, Y. Liu and G. Yang, An improved hybrid particle swarm optimization algorithm for fuzzy p-hub center problem. Comput. Ind. Eng. 64 (2013) 133-142.

[51] M. Zhalechian, S.A. Torabi and M. Mohammadi, Hub-and-spoke network design under operational and disruption risks. Transp. Res. Part E: Logistics Transp. Rev. 109 (2018) 20-43.

[52] W. Zhong, Z. Juan, F. Zong and H. Su, Hierarchical hub location model and hybrid algorithm for integration of urban and rural public transport. Int. J. Distr. Sensor Netw. 14 (2018). 